\title{
Encapsulation of Salt Hydrates by Polymer Coatings for Low- Temperature Heat Storage Applications
}

Bas G. P. van Ravensteijn, ${ }^{*} \S$ Pim A. J. Donkers, Rick C. Ruliaman, Jacco Eversdijk, Hartmut R. Fischer, Henk P. Huinink, and Olaf C. G. Adan

Cite This: ACS Appl. Polym. Mater. 2021, 3, 1712-1726

Read Online

ABSTRACT: Efficient and cheap storage of energy from renewable resources presents a key technology to facilitate the ongoing energy transition. Storing heat in thermochemical materials (TCMs), such as salt hydrates, provides a promising concept to meet this demand. TCMs can capture heat reversibly and loss-free by relying on equilibrium hydration reactions of the salts. Persistent bottlenecks in the full-scale application of this technology are the low mechanical resilience of salt grains and their tendency to coagulate or dissolve when in contact with water vapor. To overcome this, the salt grains can be encapsulated by a stabilizing
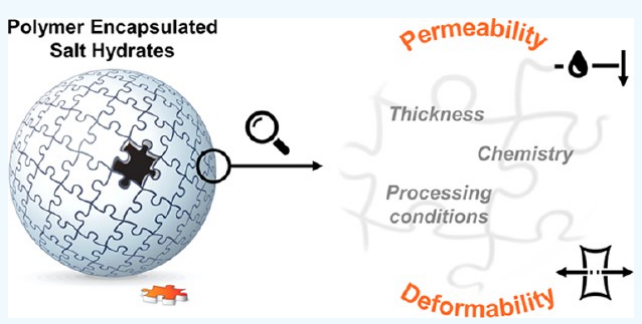
polymer coating. Ideal coatings combine high water vapor permeability with reversible deformability to minimize the resistance for water transport and to accommodate the volumetric changes of the TCM during repetitive (de)hydration, respectively. Here, a systematic study into the applicability of commercially available polymers as coating materials is presented. Mechanical analysis and wet-cup experiments on freestanding polymer films revealed that cellulosebased coatings successfully combine permeability and ductility and meet the engineering demands for domestic TCM-based heat storage applications. The validity of using freestanding films as model system was confirmed by encapsulating granular TCMs in ethyl and hydroxyl propyl cellulose using fluidized bed coating. The permeability was retained and an enhanced structural integrity of the TCM grains during (de)hydration cycles was observed.

KEYWORDS: salt hydrates, thermochemical materials, encapsulation, heat storage, core-shell

\section{INTRODUCTION}

Driven by current societal demands, an energy transition in which we move away from carbon-based to renewable energy sources fueled by solar radiation, wind energy, and hydropower has been actively initiated over the past years. ${ }^{1}$ Technological advancements increased the efficiency of capturing and utilizing the energy from these sources significantly. However, the availability for especially solar and wind energies are highly subjected to fluctuations on seasonal, daily, and even hourly time scales. Therefore, the power that can be generated from these renewable sources fluctuates, resulting in an unpredictable energy supply that is unable to meet societal energy demands reliably. To closely match energy demand and supply, energy storage systems that account for the fluctuations in energy production are therefore indispensable elements to realize the energy transition successfully.

As the majority (approximately $80 \%$ ) of the residential energy consumption in European households is used for space heating and hot tap water generation, ${ }^{2}$ a method to store carbon-free heat would have a great impact on reducing the overall carbon fingerprint. A promising concept to store sustainable heat, which is generally obtained via solar radiation, is based on a thermochemical reaction. ${ }^{3-6}$ The solid materials involved in these reactions are called thermochemical materials (TCMs). Key advantages with respect to techniques like sensible heat storage and phase change materials (PCM) include a nearly loss-free storage period and a high energy density. In general, the gas-solid equilibrium reaction responsible for the storage can be represented by

$$
\mathrm{MX} \cdot n \mathrm{~L}(\mathrm{~s}) \rightleftharpoons \mathrm{MX} \cdot m \mathrm{~L}(\mathrm{~s})+(n-m) \cdot \mathrm{L}(\mathrm{g})
$$

where $\mathrm{MX} \cdot n \mathrm{~L}(\mathrm{~s})$ is a solid salt complex consisting of a salt MX. $m \mathrm{~L}(\mathrm{~s})$ and $(n-m) \mathrm{mol}$ of sorbent $\mathrm{L}$, typically water $\left(\mathrm{H}_{2} \mathrm{O}\right)$, ammonia $\left(\mathrm{NH}_{3}\right)$, or methanol $\left(\mathrm{CH}_{3} \mathrm{OH}\right)$. The amount of reactive gas $\mathrm{L}$ inside salt complex $\mathrm{MX}$ is called the loading of the salt. The formation reaction of MX· $n \mathrm{~L}$ is exothermic, i.e., it produces energy that can be used for heating purposes. The reverse decomposition reaction of $\mathrm{MX} \cdot n \mathrm{~L}$ is endothermic, and thus costs energy. The equilibrium reaction in eq 1 implies that the maximum loading of a salt $\mathrm{MX}$ at a temperature $T$ is determined by the vapor pressure of $\mathrm{L}(\mathrm{g})$. For the practical application, the hydration and dehydration reactions are separated by the controlled supply or removal of water

Received: October 22, 2020

Accepted: March 5, 2021

Published: March 15, 2021 
vapor. Control over these (de)hydration reactions can be realized in a so-called heat battery. Although different designs are proposed in the literature, ${ }^{5}$ these reactor systems contain two compartments, one filled with a TCM and the other with water. These compartments are separated by a valve ensuring that the heat battery can be charged and discharged on demand.

As residential applications are envisioned for these heat batteries, the use of $\mathrm{H}_{2} \mathrm{O}$ as sorbent has the preference to ensure all safety considerations and regulations are met. However, the use of water introduces several material challenges that need to be overcome to successfully employ these salt-based batteries in terms of lifetime and maximum attainable storage capabilities. To promote the reaction kinetics of the hydration reaction, and therefore the power output that can be generated, these salt-based batteries require a large surface area of salt to be in contact with the water vapor. To realize this, beds of small salt granules are typically used. However, promising salts for the envisioned application, such as calcium chloride $\left(\mathrm{CaCl}_{2}\right)$, potassium carbonate $\left(\mathrm{K}_{2} \mathrm{CO}_{3}\right)$, and strontium chloride $\left(\mathrm{SrCl}_{2}\right)$, are (highly) soluble in water. $^{5}$ Exposing these salts to water vapor will insurmountably lead to a (partial) dissolution and/or fusion of salt grains during cyclic loading and unloading of the battery, with a decrease in performance as a consequence.

To overcome the dissolution and coagulation and to increase the mechanical resilience of the grains, the salts have to be stabilized. Stabilization can be achieved by embedding the TCM in inorganic porous carrier materials, ${ }^{7-14}$ such as zeolites, metal-organic frameworks, or silicates and polymer supports. ${ }^{15-21}$ Alternatively, a so-called core-shell method can be used for stabilization. In this case, the TCM is encapsulated by a polymeric or inorganic coating. ${ }^{4,22-29}$ In contrast to the porous stabilization matrices, these coatings generally have negligible volume compared to the volume of active TCM, ultimately translating into beds with higher energy storage densities (see Section S1, Supporting Information). Despite the considerable body of literature on this topic, no systematic studies were performed to relate the material properties of the shell to the performance in heat storage applications.

Here, we address this shortcoming by performing a systematic evaluation of various commercially available polymers as stabilizing shell materials. The main criteria in selecting promising shell materials are water vapor permeability and shell elasticity/ductility. A high water vapor permeability $(P)$ is required to ensure efficient transport of the reactive gas to and from the salt and therefore limit the apparent decrease in activity of the salt due to transport resistance. Naturally, the desired permeabilities depend on the final application of the heat battery. For this work, three residential case studies were considered to estimate desired target values for the water vapor permeability. Evidently, the amount of material used per day will be different depending on the type of house type, outdoor conditions, and end-user behavior:

1. Tap water without buffer: In this situation, the heat battery delivers sufficient heat to directly meet a warm water need, for example, to produce warm shower water. Assuming an average shower requires $10 \mathrm{~min}$ of $40{ }^{\circ} \mathrm{C}$ water, whereby the initial temperature of the water is 10 ${ }^{\circ} \mathrm{C}$, approximately $13 \mathrm{MJ}$ is required for this scenario. This energy can be supplied by $20 \mathrm{~kg}$ of $\mathrm{K}_{2} \mathrm{CO}_{3}$, which translates to a power output on the material level on the order of $1000 \mathrm{~W} / \mathrm{kg}$.

2. Tap water with buffer: In contrast to the unbuffered situation, a water buffer can be charged over a longer period of time. Therefore, the entire daily tap water demand (approximately $30 \mathrm{MJ}$ ) can slowly be charged in the buffer. If a recharge time of $2 \mathrm{~h}$ is assumed and 30 $\mathrm{MJ}$ is released in a daily cycle, approximately $60 \mathrm{~kg}$ of $\mathrm{K}_{2} \mathrm{CO}_{3}$ should suffice. On the material level, a power of $70 \mathrm{~W} / \mathrm{kg}$ is needed.

3. Floor heating: In this case, the building will be heated with the aid of a TCM. The TCM will dissipate all of the heat with a constant power throughout the day, i.e., a house with a constant heating demand of $6 \mathrm{~kW}$ consumes over $24 \mathrm{~h} 144 \mathrm{kWh}$ (518 MJ). To deliver this heat in a house, $790 \mathrm{~kg}$ of $\mathrm{K}_{2} \mathrm{CO}_{3}$ is needed, which translates to a power of $7 \mathrm{~W} / \mathrm{kg}$. In case of higher power demands, the amount of material scales linearly, meaning that the power on material level is independent of the final heat consumption under the current assumptions.

Based on these three use cases, the minimum required water vapor permeability was estimated according to

$$
P=\frac{\mathrm{d} Q}{\mathrm{~d} t} \frac{d}{A \Delta p}
$$

where $\mathrm{dQ} / \mathrm{d} t$ represents the water vapor transmission rate $[\mathrm{g} /$ $\mathrm{s}], d$ is the coating thickness [m], $A$ is the coating surface through which vapor evaporation takes place $\left[\mathrm{m}^{2}\right]$, and $\Delta p$ is the water vapor pressure difference over the polymeric film. The vapor transmission rate $\mathrm{d} Q / \mathrm{d} t$ is directly related to the power output per $\mathrm{kg}$ of TCM as defined for the individual use cases above. $\Delta p$ was taken to be equal to $100 \mathrm{~Pa}$. $d$ was assumed to be $10 \mu \mathrm{m}$, while $A$ is related to the radius of the salts grains, which is assumed to be $1 \mathrm{~mm}$. This grain size was selected based on a trade-off between optimizing (de)hydration kinetics while limiting the pressure drop over a bed of grains in the final application. As the optimal grain size might deviate, the sensitivity of the permeability on grain size was calculated (Section S2, Supporting Information). Given the relatively weak dependence between the particle radius and required permeability, the results and conclusions discussed below are valid within a reasonable window of grain sizes. Based on the input values listed above, minimal water vapor permeabilities were found for the different use cases (Table 1).

In addition to the demand for a high permeability, the polymer encapsulating shell should be deformable. This requirement originates from the fact that salts expand (20$50 \%)$ upon hydration. ${ }^{30}$ Flexible outer shells will be able to accommodate this volumetric change without failure. Prehydration of the salts could be considered such that coatings can

Table 1. Minimal Water Vapor Permeability $(P)$ of an Encapsulating Coating with a Thickness of $10 \mu \mathrm{m}$ on TCM Grains with a $1 \mathrm{~mm}$ Radius to Reach the Demanded Power Output of a Salt Hydrate-Based Heat Battery for Three Residential Use Cases use case tap water with buffer

floor heating tap water without buffer 
be applied on already expanded salts. However, the detrimental loss in structural integrity of the active material makes subsequent coating via, for example, fluidized bed coating, no longer possible. In addition, when considering the production of coated materials on industrial scales, the energy required to pretreat the salt would be unacceptably high.

With these design criteria in mind, the applicability of a variety of commercially available polymers as encapsulating materials for salt-based TCMs was screened. Readily available polymers were used to maximize the relevance of this study for real-life heat storage applications. Screening was performed using tensile tests and wet-cup permeability measurements on macroscopic films with well-defined thicknesses and process history. The effect of coating thickness, molecular weight (MW), casting solvent, and cross-link density was investigated to fully optimize the coating performance. Ethyl and hydroxypropyl cellulose-based coatings combine permeabilities and mechanical properties compatible with low-power residential use cases for TCM-based heat storage. By translating this coating formulation to processing conditions for industrially relevant fluidized bed encapsulation of TCM granules, a retainment in water vapor permeability and enhanced mechanical resilience during repetitive (de)hydration cycles could be realized.

\section{EXPERIMENTAL SECTION}

2.1. Materials. Ethyl cellulose (EC, 10, 46, 100, $300 \mathrm{cP} 5 \%$ in toluene/ethanol (80:20), 48\% ethoxyl), methyl cellulose (MC, $400 \mathrm{cP}$ 2 wt $\%$ in water at $20{ }^{\circ} \mathrm{C}$ ), hydroxypropyl cellulose (HPC, MW 100 000), poly(vinyl alcohol) (PVA100, MW 130000 , degree of hydrolysis $>99 \%)$, Disperse Orange 3 (DO3, dye content $=90 \%)$, methylene blue hydrate $(\mathrm{MB}, \geq 95 \%)$, hexamethylene diisocyanate (HMDI, purum, $\geq 98 \%$ ), and molecular sieves (5 $\AA$, beads $8-12$ mesh, $\left.\mathrm{Ca} /{ }_{n} \mathrm{Na}_{12-2 n}\left[\left(\mathrm{AlO}_{2}\right)_{12}\left(\mathrm{SiO}_{2}\right)_{12}\right] \cdot x \mathrm{H}_{2} \mathrm{O}\right)$ were obtained from Sigma-Aldrich. Poly(vinyl alcohol) (PVA18-88, MW 130 000, degree of hydrolysis $=86.7-88.7 \%$ ) was purchased from Fluka. Dichloromethane (DCM, $\geq 99.9 \%$ ) was obtained from BioSolve. Potassium carbonate powder $\left(\mathrm{K}_{2} \mathrm{CO}_{3} \cdot 1.5 \mathrm{H}_{2} \mathrm{O}, 83-85 \%\right.$ hydrated from Evonik was used for the wet-cup experiments. For fluidized bed coating, $\mathrm{K}_{2} \mathrm{CO}_{3} \cdot 1.5 \mathrm{H}_{2} \mathrm{O}$ beads $(98.5 \%, 1-2 \mathrm{~mm})$ from Fisher Scientific and carbon binder containing $\mathrm{K}_{2} \mathrm{CO}_{3}$ granules developed in the EU 2020 CREATE Project ${ }^{31}$ and produced by Caldic B.V. ${ }^{32}$ were used. All chemicals were used as received. The water used in all experiments was purified with a Milli-Q purification system (resistivity $=18.2 \mathrm{M} \Omega \cdot \mathrm{cm}$ at $25^{\circ} \mathrm{C}$ ).

2.2. Preparation of Polymer Solutions. HPC (4 wt \%), EC10 (15 wt \%), EC46 (7.5 wt \%), EC100 (7.5 wt \%), and EC300 (4 wt \%) solutions were obtained by adding the solid polymers to DCM while stirring vigorously. After addition, the samples were stored on a roller table to ensure complete dissolution. Regardless of the molecular weight of the polymers, slightly yellow, transparent solutions were obtained. An aqueous MC solution (3 wt \%) was prepared in two steps. First, one-third of the total required volume of water was heated to $80{ }^{\circ} \mathrm{C}$. The dry MC powder was added under vigorous stirring. Stirring was continued until the polymer particles were thoroughly wetted and evenly dispersed, yielding a white dispersion. In the second step, complete solubilization of the MC was achieved by adding the remaining two-thirds of the water as ice to the hot MC dispersion. The resulting solution was kept at $0-5{ }^{\circ} \mathrm{C}$, finally yielding a clear and colorless solution. A PVA18-88 solution (10 wt \%) was obtained by adding the polymers to the required volume of water under vigorous stirring. The resulting solvent swollen polymer aggregates were fully dissolved by heating the solution to $80^{\circ} \mathrm{C}$ for $15 \mathrm{~min}$, resulting in a clear and homogeneous solution. PVA100-based solutions (7.5 wt \%) were prepared by slowly adding the solid polymer to hot water $\left(90-95^{\circ} \mathrm{C}\right)$ under vigorous stirring. Directly before use, the polymer solutions were centrifuged ( $5 \mathrm{~min}, 12800 \mathrm{~g}$ ) to minimize the presence of (solid) impurities and/or air bubbles. The reported polymer concentrations yielded solutions with viscosities that facilitated the fabrication of freestanding polymer films as described in Section 2.3. In case lower-viscosity solutions were required, e.g., for the fluidized bed coating (Section 2.5), the reported solutions were diluted by addition of extra solvent. Subsequently, the solutions were left to homogenize on a roller table overnight before use.

2.3. Preparation of Freestanding Polymer Films. A glass plate $(20 \times 30 \mathrm{~cm})$ was thoroughly cleaned with water and soap and dried with dust-free paper. The plate was placed on a horizontal surface and a few milliliters of water were deposited on the plate. A sheet of tin (Sn) foil (thickness $=9 \mu \mathrm{m}$ ) was placed on top of the wetted glass plate, and with the aid of a rubber roller, the foil was evened out to form a smooth coating surface. After removing excess tin foil, tape (thickness $=50 \mu \mathrm{m}$ ) is placed along the long sides of the glass. This tape will facilitate the sliding of the coating knife without damaging the fin foil during film application. Next, a coating knife with the desired gap dimensions $(150-750 \mu \mathrm{m})$ is placed on the tape at one end of the tin foil-covered glass plate. A solution containing the target polymer $(30-40 \mathrm{~mL}$, as prepared in Section 2.2$)$ was carefully poured in front of the coating knife. A wet film was formed by smoothly pulling the coating knife over the glass plate. The obtained films were left to dry under ambient conditions for at least $24 \mathrm{~h}$. Representative pictures of the process can be found in Section S3, Supporting Information. After complete drying, the polymer-coated tin foil was removed from the glass plate. To obtain freestanding polymer films, the tin substrate was removed by dissolution in mercury. To this end, the polymer-coated tin foil was placed with its tin side down on a layer of mercury. After $2-3 \mathrm{~h}$, the resulting freestanding polymers films were removed from the mercury bath and cleaned from residual tin/mercury with a brush and paper towels. The average film thickness was determined, and the films were stored in plastic sleeves to prevent any damage.

2.4. Preparation of Cross-Linked EC Films. The procedure toward cross-linked EC100 and EC300 coatings was similar to the method described in Section 2.3 for non-cross-linked films. Cross-link densities, here defined as the percentage of EC's hydroxyl groups that will react with the cross-linker (see Section S4 (Supporting Information) for calculation), of 1,10 , and $100 \%$ were targeted. To achieve this, HMDI $(18,180$, or $1800 \mu \mathrm{L})$ dissolved in DCM $(1 \mathrm{~mL})$ was added to the DCM-based EC100 or EC300 solution (40 g) directly before use. The resulting solution was vortexed to ensure a homogeneous distribution of HMDI and subsequently centrifuged (5 min, $12800 \mathrm{~g}$ ) to remove introduced air bubbles. This centrifuged solution was used for film formation. After a dried polymer film was obtained, the extent of cross-linking was monitored using infrared (IR) spectroscopy by following the isocyanate-related vibration at $2260 \mathrm{~cm}^{-1}$.

2.5. Coating of Zeolite and $\mathrm{K}_{2} \mathrm{CO}_{3}$ Beads. Zeolite and $\mathrm{K}_{2} \mathrm{CO}_{3}$ granules were encapsulated with an EC, HPC, or PVA18-88 layer by fluidized bed coating. The coater (ProCept 4M8-TriX) was operated in bottom spraying mode with the nozzle $(0.6 \mathrm{~mm})$ supplying the carrier gas and the polymer solution positioned at the bottom of the coating vessel. $\mathrm{K}_{2} \mathrm{CO}_{3}(200 \mathrm{~g}, 1-2 \mathrm{~mm}$ grains $)$ or zeolite beads $(150-250 \mathrm{~g})$ were introduced in the $1 \mathrm{~L}$ vessel. A fluid bed of these granules was generated by supplying an air flow through the nozzle (see Table S2 for operating settings). The temperature of the bed was set by supplying hot air into the coating vessel. Once a stable bed was formed in terms of fluidization and temperature, the polymer solution was introduced into the reaction vessel. The solution was supplied with a peristaltic pump to the atomization nozzle (see Table S2 for addition rates). Polymer concentrations were chosen such that the zero-shear viscosities $\left(\eta_{0}\right)$ were equal to $30-60 \mathrm{mPa} \cdot \mathrm{s}$. The quantity of solution added to the fluidized bed was determined by placing the solution reservoir on a balance. The employed polymer solutions were stained with a dye $(1 \mathrm{mg} / \mathrm{mL}, \mathrm{DO} 3$ for DCM-based and MB for water-based polymer solutions) to visually monitor the formation of the polymeric shell during the coating process. Samples were withdrawn during the procedure to probe the shell thickness, 
(a)

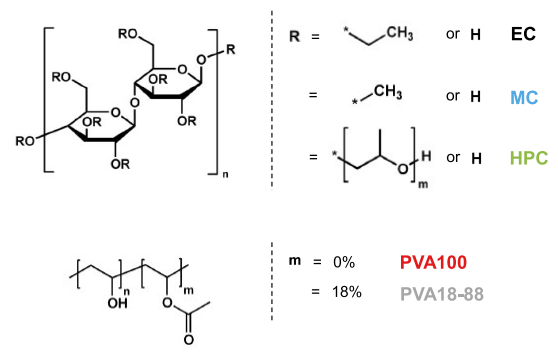

(b)

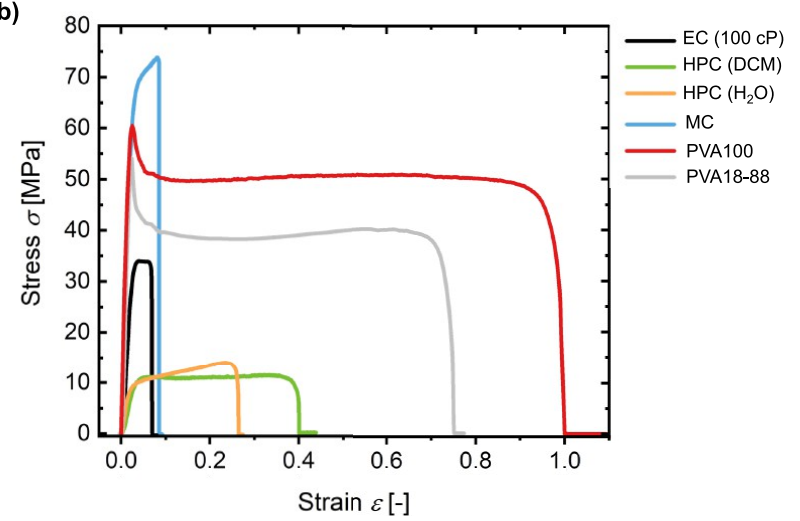

Figure 1. (a) Chemical structures of applied polymers and their abbreviations: $\mathrm{EC}=$ ethyl cellulose (black), MC = methyl cellulose (blue), HPC = hydroxypropyl cellulose (green, orange), PVA = poly(vinyl alcohol) (red, gray). (b) Stress $(\sigma)-$ strain $(\varepsilon)$ curves obtained for the thin films composed of the polymers depicted in (a).

morphology, and water vapor permeability as a function of time. The encapsulated beads were stored in a controlled environment $(T=20$ ${ }^{\circ} \mathrm{C}$, relative humidity $\left.(\mathrm{RH})=20 \%\right)$.

2.6. Cyclic (De)hydration of Coated $\mathrm{K}_{2} \mathrm{CO}_{3}$ Beads. For cyclic stability tests on a larger scale $(>100 \mathrm{mg})$, a small stainless-steel reactor (diameter $=68 \mathrm{~mm}$, height $=120 \mathrm{~mm}$ ) was used. During experiments, this vessel was filled with TCM and contained between 400 and $600 \mathrm{~g}$ of active material (HPC-coated $\mathrm{K}_{2} \mathrm{CO}_{3}$ grains with binder). The vessel was isolated at the walls, and a controlled air flow was supplied to the vessel for hydration or dehydration reactions. The air flow conditions are controlled with a condenser/humidifier of Permapure (FC400-2500-7LP/HP) and an air heater of Tucto (Flow Torch 200). The following cyclic hydration/dehydration conditions were applied: hydration: $30^{\circ} \mathrm{C}$ at $14 \mathrm{mbar}$ for $20 \mathrm{~h}$ at an air flow of 6 $\mathrm{m}^{3}$ air/h; dehydration: $120^{\circ} \mathrm{C}$ at $14 \mathrm{mbar}$ for $5 \mathrm{~h}$ at an air flow of 9 $\mathrm{m}^{3}$ air/h. These conditions were measured at the inlet of the vessel. The outlet conditions are determined by the response of the bed. The conditions were chosen such that deliquescence of the $\mathrm{K}_{2} \mathrm{CO}_{3}$ particles was avoided.

2.7. Characterization. The average film thickness of the freestanding polymer films was calculated from thicknesses measured at six positions (four corners and two positions in the center) using a Mitutoyo micrometer.

Mechanical tests were performed using a TesT $110.2 \mathrm{kN} \cdot \mathrm{h}$ tensile tester. Dog bone-shaped specimens (sample area: $30 \times 7 \mathrm{~mm}$ ) were prepared using a custom-made die cutter (Behrens Stanztechnik). Before measuring, the average thickness of the thin section of the dog bone was evaluated from three measurements with a micrometer. Dedicated clamps lined with rubber inserts were used to fixate the thin samples in the mechanical tester to avoid fallacious rupture at the clamps (see Section S6, Supporting Information). The samples were subjected to a constant pulling speed of $0.01 \mathrm{~mm} / \mathrm{s}$, and the force required to deform the samples was measured. Using the average thickness of the dog bone sample and its initial dimensions, the raw data were converted to stress $(\sigma)$-strain $(\varepsilon)$ curves. For each coating, a set of five to seven mechanical tests were performed to confirm the reproducibility of the results.

Water vapor permeabilities were determined using wet-cup experiments. To this end, circular-shaped specimens $(\varnothing=30 \mathrm{~mm})$ were cut from the freestanding polymer films. These films were fixated on top of $50 \mathrm{~mL}$ vials containing a saturated $\mathrm{K}_{2} \mathrm{CO}_{3}$ solution $(20-30$ $\mathrm{mL}$ ) using ethyl cyanoacrylate-based glue (Loctite Super Glue Gel Control). To promote the adherence of the film, the glass surface in contact with the polymer film was treated with sandpaper. In addition to the glue, the interface between the glass and the polymer was sealed using bee wax. The vials were placed in a controlled environment $\left(T=20^{\circ} \mathrm{C}, \mathrm{RH}=20 \%\right)$. The water vapor permeabilities were determined by following the decrease in mass over time. These data served as input to calculate the water vapor permeability $(P)$ of the coatings as described by eq 2 . The reported permeabilities were calculated after monitoring the samples for 50 days. These long measurement times were required to ensure steady-state permeabilities were obtained (see Section S7 (Supporting Information) for $P$ as a function of time). For each coating, at least five individual wetcup experiments were performed to evaluate the reproducibility of the measured permeabilities and account for potential defects in the polymer films.

Infrared (IR) spectra of the polymer films were recorded using a Thermo Scientific Nicolet 6700 spectrometer operating in attenuated total reflection (ATR) mode.

Zero-shear viscosities $\left(\eta_{0}\right)$ were measured using an Anton Paar rolling ball microviscometer equipped with a glass capillary $(\varnothing=1.8$ $\mathrm{mm})$ and gold-plated ball $(\varnothing=1.5 \mathrm{~mm})$. The measurements were performed at $25{ }^{\circ} \mathrm{C}$ and a tilt angle of $70^{\circ}$. The viscosities were calculated based on the average rolling time from six measurements.

Optical microscopy images were recorded with a Keyence VHX600 digital microscope. To visualize the coating thickness, crosssectional images were made after a coated granule was bisected with a sharp razor blade.

Scanning electron microscopy (SEM) analysis was performed using an FEI Quanta 600. A gaseous secondary electron detector (GSED) from FEI was used with an aperture of $500 \mu \mathrm{m}$. This detector allows a pressure range between 0.3 and $27 \mathrm{mbar}$ in the specimen chamber with an accuracy of 0.1 mbar. However, for the experiments in this study, sufficient image quality was obtained for pressures of approximately 4 mbar.

The rate of water uptake of a single particle was tested using a thermogravimetric analyzer (TGA) of type Mettler-Toledo TGA/ SDTA 851e. The oven was purged with air $(300 \mathrm{~mL} / \mathrm{min})$ with a relative humidity of $50 \%$ at $21^{\circ} \mathrm{C}$, corresponding to a constant partial water vapor pressure of $12 \mathrm{mbar}$ inside the oven. The samples were tested using the following temperature profile: equilibration at $92{ }^{\circ} \mathrm{C}$ $(120 \mathrm{~min})$, dehydration at $37^{\circ} \mathrm{C}(6 \mathrm{~h})$, with a cooling rate of $10^{\circ} \mathrm{C} /$ min. The equilibration step prior to hydration is necessary to guarantee stable starting conditions because the samples might partially hydrate during their preparation.

\section{RESULTS AND DISCUSSION}

3.1. Effect of the Polymer Chemical Composition. As starting point for the evaluation of suitable polymers to stabilize salt hydrates in a core-shell morphology, a selection of commonly applied and commercially available polymers was made. The chemical structures of the used polymers are depicted in Figure 1. The selection was inspired on extensive pharmaceutical literature, where the listed polymers are frequently used to fabricate polymeric coatings with controlled barrier properties for pharmaceutically active molecules. ${ }^{33-39}$ In addition, these polymers are produced at large scales, 
making them economically attractive candidates for mass production of encapsulated TCMs. For this study, poly(vinyl alcohol) (PVA) with degrees of hydrolysis (percentage of acetate groups hydrolyzed to the alcohol) equal to $100 \%$ (PVA100) and 88\% (PVA18-88) and modified cellulose derivates, namely, ethyl cellulose (EC), methyl cellulose (MC), and hydroxypropyl cellulose (HPC), were used. The PVA-based coatings were cast from aqueous solutions, as these polymers are (highly) hydrophilic. The hydrophilicity of the cellulose-derived polymers is tunable with the choice of pendant modification group (Figure $1 \mathrm{a}, \mathrm{R}$ ) and decreases when going from MC to HPC and finally EC. MC-based coatings were cast from water, while for EC, dichloromethane (DCM) was used as a solvent. HPC films were prepared from either DCM or water-based polymer solutions.

To gain fundamental insight into how the polymer properties influence the stabilizing capability of salt hydrates for heat storage applications, elementary mechanical properties and permeabilities for water vapor were initially determined for freestanding polymer films. The freestanding films can be fabricated in a controlled manner yielding defect-free films with homogeneous thicknesses (see Sections 2.3 and S3). These model coatings do therefore serve as a benchmark for the properties of polymeric films, and their use eliminates the hard-to-quantify effects of processing conditions on coating characteristics when relying on (industrially relevant) techniques to coat salt grains with a polymeric layer, e.g., spray drying or fluidized bed coating (see Section 3.6).

Stress $(\sigma)$-strain $(\varepsilon)$ curves of the set of polymers are depicted in Figure 1b. Coatings with comparable thicknesses $(20-30 \mu \mathrm{m})$ were prepared to minimize the influence of this parameter on the comparison between the different coating materials. Although the coating thickness has an effect on the mechanical properties (see Section 3.5), the variation among this set of coatings is not expected to alter the observed trends. The curves were obtained using a constant deformation rate of $0.01 \mathrm{~mm} / \mathrm{s}$, inspired by the rates of deformation that coatings applied on salts encounter during hydration (see Section S8, Supporting Information). Evidently, the mechanical properties of the selected polymers vary significantly, both in maximum achievable strains $\left(\varepsilon_{\max }\right)$ and yield stresses $\left(\sigma_{\mathrm{y}}\right)$. The maximum stress that these polymeric films can withstand varies between approximately 10 and $75 \mathrm{MPa}$. These values are not sufficient to counteract the stresses imposed during hydration of the salt. Steiger performed a calculation of crystallization pressures $\left(p_{\text {cryst }}\right)$ on salt hydrates and showed that pressures on the order of $1-20 \mathrm{MPa}$ can be expected during hydration of a salt. ${ }^{40}$ Considering spherical TCM grains with a radius $(r)$ and a homogeneous surface coating with a thickness $(\delta)$ of $10 \mu \mathrm{m}$, the stress imposed on this polymer film is given by eq 3 , which is on the order of $1 \mathrm{GPa}$.

$$
\sigma_{\min }=\frac{p_{\text {cryst }} r}{2 \delta}
$$

Evidently, the required stresses are orders of magnitude larger than the yield points of all our coatings. For these films to act as successful encapsulating materials, the shell needs to be able to accommodate the volumetric expansion and not sustain the pressure induced by the hydrating salt. In this respect, the PVA-based films (Figure $1 \mathrm{~b}$, red and gray curves) are promising candidates, as $\varepsilon_{\max }$ of $80-100 \%$ could be attained. The tensile curves are characterized by a pronounced yield point $\left(\sigma_{\mathrm{y}}\right)$ separating the elastic from the plastic regime. The majority of the deformation takes place in the plastic regime, implying that the deformations are not reversible. Fully reversible or elastic behavior is, in principle, not required as long as the coating is capable of deforming up to the maximum dimensions of the hydrated salt, while remaining intact upon contraction of the salt grain during dehydration. For the cellulose-based films, $\varepsilon_{\max }$ varies between 10 and $40 \%$ depending on the chemistry of the pendant groups, where the HPC derivatives are able to accommodate the highest strains.

As mentioned in Section 1, the mechanical characteristics of the films are not the only selection criterium. The water vapor permeability is of great importance as well since this property will have a significant influence on the rate of (de)hydration and hence the power output of the heat battery as a whole. The water vapor permeabilities for the different polymer films as measured using so-called wet-cup experiments are shown in Figure 2. To exclude the influence of coating thickness on the

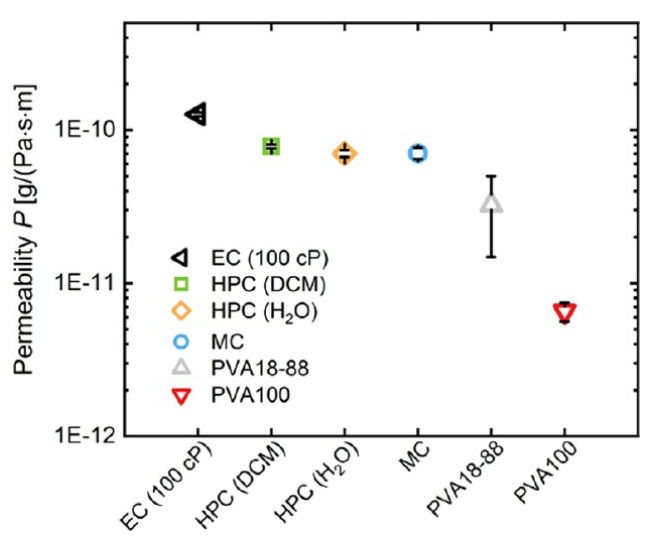

Figure 2. Water vapor permeabilities $(P)$ derived from wet-cup experiments for cellulose- and poly(vinyl alcohol)-based films. The permeabilities were calculated after monitoring the weight loss due to evaporation for 50 days. The errors on the permeabilities were calculated from five individual wet-cup experiments per film.

permeability, for example, due to thickness-dependent microstructural variations (see Section 3.5), polymer films of similar thicknesses $(20-30 \mu \mathrm{m})$ were used for this analysis. The measured permeabilities vary over an order of magnitude from $1.3 \times 10^{-10}$ to $7 \times 10^{-12} \mathrm{~g} /(\mathrm{Pa} \cdot \mathrm{s} \cdot \mathrm{m})$ and are in agreement with previously reported values for pure, homogeneous polymer films. ${ }^{41-45}$ The water vapor permeability increases with increasing hydrophobic character of the polymer film. This trend can be explained by the absence or limited interactions of the water molecules with the apolar polymers. The higher polarity and hydrogen-bonding capabilities of the PVA-based coatings promote higher residence times of the water in polymeric films, effectively slowing down the water transport. An additional contribution to the limited water vapor permeability for PVA-based matrices is related to the crystallinity of these polymers. Crystallization leads to domains with tightly packed and rather immobile polymer chains, which hamper efficient transport of water molecules. The cellulosederived films are completely amorphous, ensuring maximum chain mobility and hence a minimal barrier against water permeation. Without further optimization, the permeabilities of the cellulose-based coatings are on the appropriate order or magnitude to facilitate the application scenarios for salt-based heat storage outlined in Section 1. Coatings based on PVA 
seem too impermeable for water vapor, and therefore not suitable.

Combining the results from the tensile testing (Figure 1) and the water vapor permeability data (Figure 2), EC and $\mathrm{HPC}$ are promising as successful encapsulating materials for salt hydrates. Both mechanical properties and permeability are closest to the set requirements. Despite the favorable mechanical properties of the PVA-based films, their low permeability is a significant disadvantage compared to the cellulose derivatives. As more experimental opportunities are available to tune and improve the mechanical characteristics of the polymer film compared to controlling an intrinsic property such as the water vapor permeability, the remainder of this study focuses on cellulose-based coatings. For this class of coatings, we investigate which experimental parameters can be tuned to optimally tailor the film properties for encapsulation of salt hydrates.

3.2. Effect of Molecular Weight. The first experimental parameter that was investigated was the molecular weight of the employed EC polymers. Four commercial grades of EC were used, namely, 10, 46, 100, and $300 \mathrm{cP}$. The grades are labeled according to their viscosity when dissolved in an 80:20 toluene/ethanol mixture at a concentration of $5 \mathrm{wt} \%$ (standard literature procedure). ${ }^{46}$ As polymers with higher molecular weights contribute more to the solution viscosity, increased viscosity values at a fixed concentration indicate higher molecular weights. The molecular weights corresponding to the 10,46 , and $100 \mathrm{cP}$ were previously estimated by Inukai et al. who found values of 73,140 , and $180 \mathrm{~kg} / \mathrm{mol}$, respectively, with polydispersity indices $\left(M_{\mathrm{w}} / M_{\mathrm{n}}\right)$ on the order of $3 .^{47}$

Freestanding films were prepared, and the mechanical properties and water vapor permeability were determined as outlined in Section 3.1. The results are summarized in Figure 3. Varying the molecular weight has a significant influence on the mechanical characteristic of the produced films (Figure 3a,b). ${ }^{48}$ Films comprising $10 \mathrm{cP}$ EC were extremely brittle and difficult to manipulate. This behavior translates into low ultimate strengths $(10 \mathrm{MPa})$ and low $\varepsilon_{\max }(0.01)$. Upon increasing the molecular weight of the polymers, the maximum strength and elongation that the films could accommodate increased. The $\varepsilon_{\max }$ value increased by 2 orders of magnitude going from 10 to $300 \mathrm{cP} \mathrm{EC} \mathrm{(Figure} \mathrm{3b),} \mathrm{while} \mathrm{the} \mathrm{maximum/}$ yield stress increased by a factor of 4 from approximately 10 to $40 \mathrm{MPa}$. This significant increase in deformability can be explained by two mechanisms. First, using higher-molecularweight polymers, the chances of forming chain entanglements between the polymer chains increase. These entanglements act as physical cross-links, causing the stresses imposed during tensile testing to be distributed over a larger fraction of the sample. The presence of these anticipated cross-links is reflected by an increase in tensile strength (Figure 3a), implying more energy is required to deform the films. Second, by increasing the molecular weight, the degree of crystallinity of the polymer matrix might decrease. ${ }^{49}$ Longer polymers are more difficult to pack into organized crystalline domains due to their slower chain dynamics. Since crystallites principally act as brittle/stiff domains, which are susceptible to fracture, suppressing their formation increases the ductility and deformability of the solid polymer film.

Despite the pronounced changes in mechanical properties, the water vapor permeability is practically unaffected by changes to the molecular weight (Figure 3c). For the intended (a)
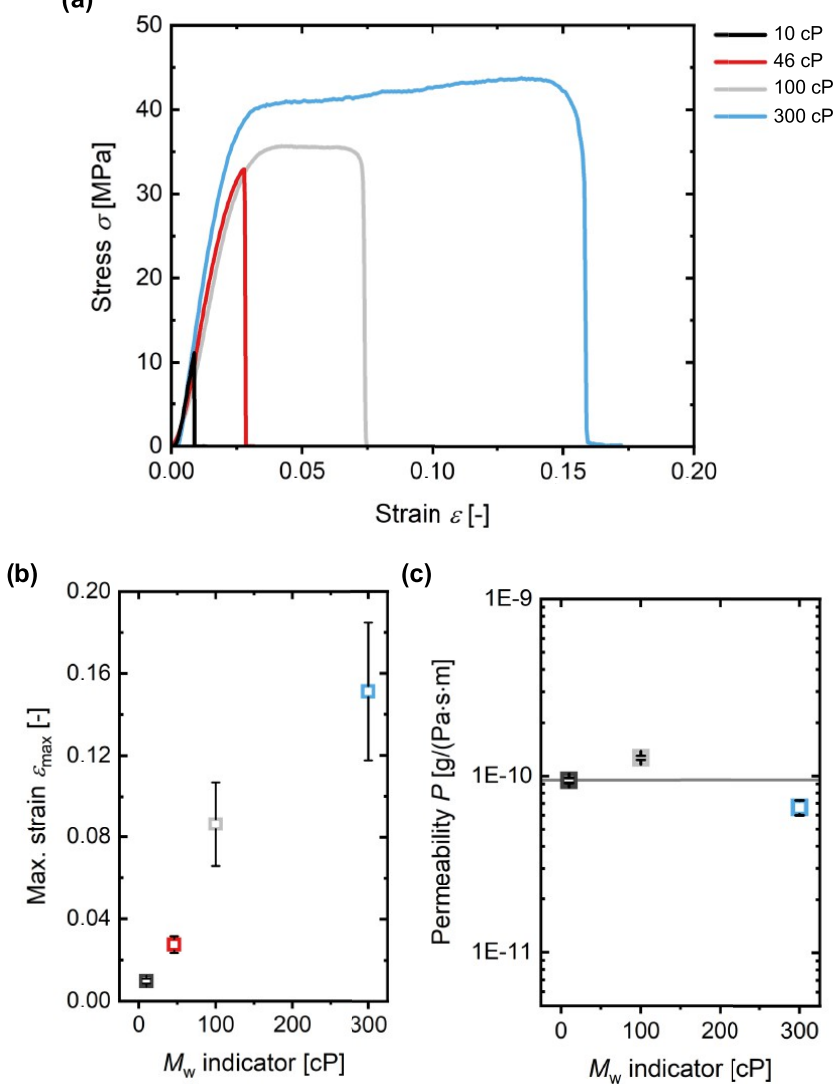

Figure 3. (a) Stress $(\sigma)$-strain $(\varepsilon)$ curves obtained for freestanding films composed of 10 (black), 46 (red), 100 (gray), and 300 (blue) cP-grade ethyl cellulose (EC). (b) Maximum attainable strain $\left(\varepsilon_{\max }\right)$ and $(c)$ water vapor permeabilities $(P)$ derived from wet-cup experiments for EC films as a function of molecular weight.

use as a stabilizing material for salt hydrates, using highmolecular-weight polymers is therefore highly recommended since these materials significantly increase the maximum attainable deformability, while no compromise is required on the water vapor permeability.

3.3. Effect of Chemical Cross-Linking. Cross-linking the individual polymer chains together is expected to have a distinct influence on the mechanical properties of the freestanding films. As elaborated in the previous section, introducing cross-links to the polymeric material prevents individual polymer chains to slide along each other, thereby potentially introducing elastic character to the polymeric matrix instead of plasticity. In addition to physical cross-link points caused by chain entanglements, cross-links can also be introduced chemically by covalently linking individual polymer chains. For the EC polymers, a diisocyanate cross-linking chemistry was employed (Figure $4 a) .{ }^{50}$ Cross-linking occurs as unsubstituted primary hydroxyl groups of the cellulose polymers react with the isocyanate $(-\mathrm{NCO})$ moieties to form urethane linkages. Since the cross-linkers have two isocyanate functionalities, individual polymers can be tethered together. Cross-linking was achieved by simply adding the diisocyanates to the casting solutions immediately before film fabrication.

The cross-linking reactions were performed using 100 and $300 \mathrm{cP}$-grade EC with cross-link densities of 1, 10, and 100\% with respect to the theoretical number of hydroxyl moieties present on the polymers. This theoretical number of functional 
(a)
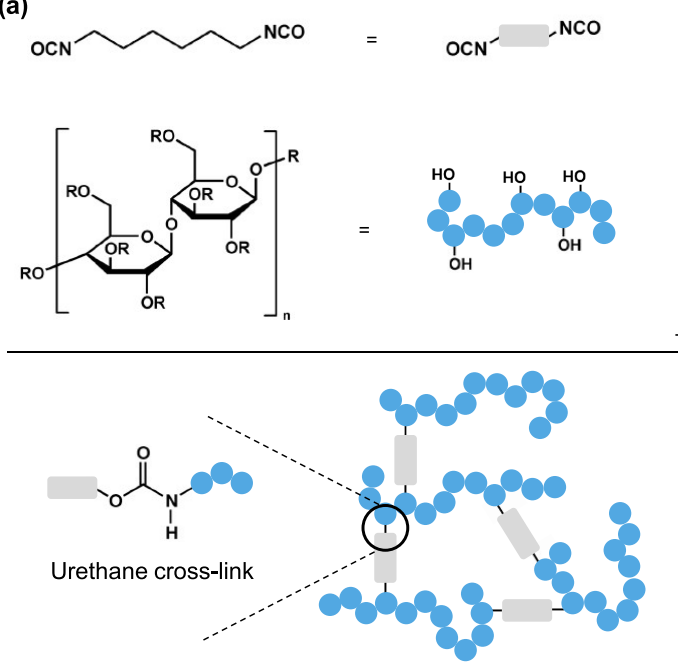

(b)

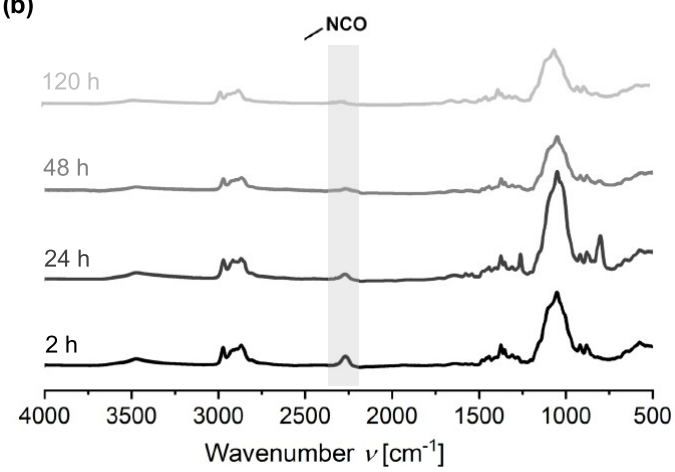

Figure 4. (a) Schematic representation of the chemical cross-linking of cellulose derivatives (blue) using diisocyanates (gray). Reaction of the hydroxyl groups $(-\mathrm{OH})$ on the polymer with the isocyanates gives rise to urethane cross-links between individual polymer chains. (b) Infrared (IR) spectra of the cross-linking of ethyl cellulose (EC, $100 \mathrm{cP}$ ) film as a function of time. The extent of cross-linking was monitored using the highlighted isocyanate-related vibrational band.

groups was calculated based on the ethoxy content of $48 \%$ ( $48 \%$ of all $\mathrm{R}$ groups in the chemical structure depicted in Figure $4 \mathrm{a}$ are $\mathrm{C}_{2} \mathrm{H}_{5}$ ). The cross-linking reaction could be easily monitored as a function of time using infrared (IR) spectroscopy as depicted in Figure $4 \mathrm{~b}$. For this exemplary spectrum, $100 \mathrm{cP}$ EC was used and a cross-link density of $10 \%$ was targeted. Clearly, the isocyanate-related vibration at approximately $2260 \mathrm{~cm}^{-1}$ decreases over time, indicative of the disappearance of the cross-linker and hence the formation of the targeted urethane linkages between the cellulose polymers. Targeting cross-link densities of 1 and $100 \%$ gave analogous spectral results (see Section S9, Supporting Information). Despite the high reactivity of isocyanates with (primary) alcohols, curing times of $120 \mathrm{~h}$ were required to reach high isocyanate conversions. The need for these long reaction times is probably related to the high viscosity and related limited chain mobility of the polymers in the film after the initial evaporation of the solvent. In addition to the IR data, successful cross-linking was confirmed by placing a small sample of cured coating into dichloromethane (DCM), a good solvent for ethyl cellulose. Regardless of the cross-link density, the films did not dissolve, strongly suggesting the formation of a covalent polymer network. In sharp contrast, a film composed of non-cross-linked EC disintegrated and dissolved in DCM instantaneously. Determining the actual degree of cross-linking in the resulting films was not attempted. The limited spectral resolution of IR spectroscopy combined with the fact that an unknown fraction of the isocyanate moieties undoubtedly reacted with water from the atmosphere during curing made any estimation highly unreliable. Fabricating the coatings under an inert atmosphere to limit side reactions and the use of more advanced analytical techniques, e.g., X-ray microscopy or atomic force microscopy (AFM), are required to control and elucidate the actual cross-link density. ${ }^{51}$ These more sophisticated experimental/analytical tools are outside the scope of this work and not required to provide an initial perception for the effect of cross-link density of the mechanics and permeabilities of the resulting films.

With the cross-linked EC coatings in hand, the mechanical properties were again evaluated using tensile testing. The results are summarized in Figure 5a,b for 100 and $300 \mathrm{cP}$ ECbased film, respectively. For the $100 \mathrm{cP}$-based coatings, using cross-link densities of $1-10 \%$ led to an increase in $\varepsilon_{\max }$ of 0.18 and 0.11 compared to 0.08 obtained for the non-cross-linked counterpart. Accompanied with this increase in maximum attainable strains was a slight increase in tensile strength and elastic modulus, representative of the increased resistance of the material against deformations. Increasing the cross-link density even further to $100 \%$ diminished the elastic character and instead caused an embrittlement and a significant increase in the strength of the film. In contrast to the results depicted in Figure 5a for the $100 \mathrm{cP}$ EC coating, cross-linking $300 \mathrm{cP}$ EC at moderate densities of $1-10 \%$ led to films, which could accommodate lower strains compared to the non-cross-linked coating. No significant difference in tensile behavior was observed when increasing the cross-link density from 1 to $10 \%$.

Considering the dependence of the molecular weight on the effect of cross-linking, we hypothesize that the major contribution cross-linking has in altering the mechanical properties is suppressing the degree of crystallization during curing. ${ }^{49,52}$ Upon cross-linking, the effective molecular weight increases and hence the polymer chain mobility decreases significantly. This suppresses the formation of crystalline domains and results in a more amorphous polymer matrix, which can accommodate larger strains before failure. As the anticipated degree of crystallinity for the $100 \mathrm{cP}$ EC is higher compared to the $300 \mathrm{cP}$ grade, the effect of crystallization suppression is more pronounced for the lower-molecularweight-based films. Since the degree of crystallization cannot be decreased further, as is the case for the highest cross-link density for the $100 \mathrm{cP}$ films and for all probed cross-link densities of the $300 \mathrm{cP}$ coatings, cross-linking causes a stiffening of the material leading to higher yield strengths and lower values of $\varepsilon_{\max }$.

As the water vapor permeability does not depend on the cross-link density (Figure 5c), cross-linking is a viable route to increase the maximum strains the coatings can sustain before failure. However, since suppression of crystallization is thought as the primary mechanism governing the enhancement of the mechanical properties, this route is only applicable for coatings based on lower-molecular-weight polymers that have a higher tendency to crystallize. When targeting high-strain-resistant films, low-cross-link densities should be targeted to prevent excessive stiffening of the polymer matrix. Since $\varepsilon_{\max }$ of the chemically cross-linked coatings could not exceed that of the coatings composed of the non-cross-linked $300 \mathrm{cP}$ EC, using high-molecular-weight polymers has the preference for the 


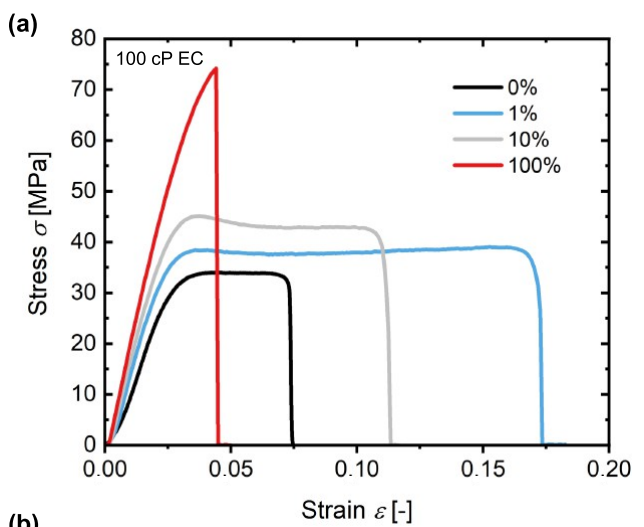

(b)
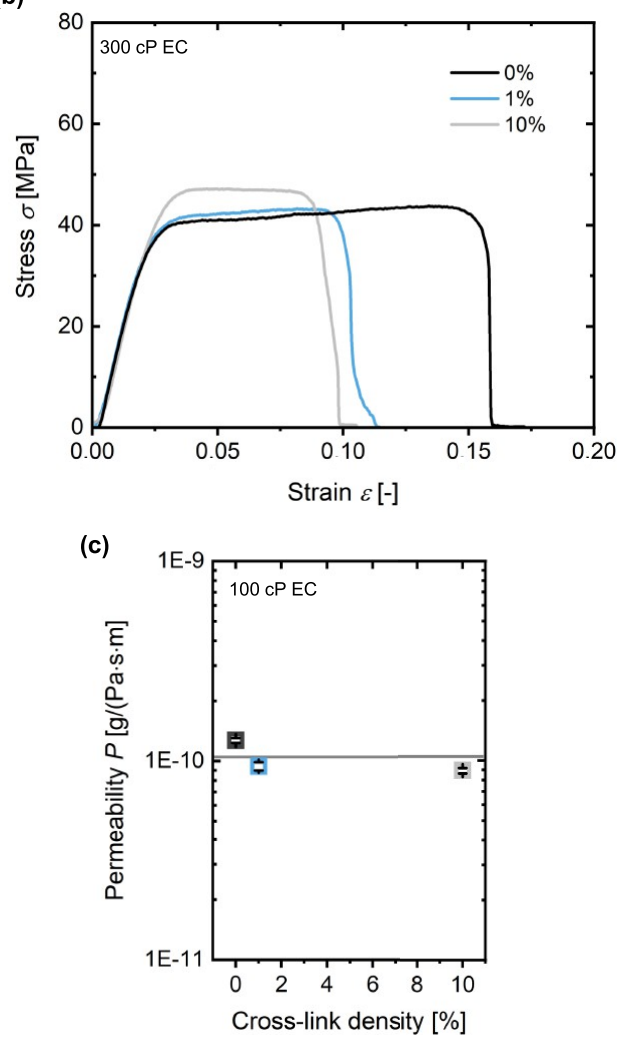

Figure 5. Stress $(\sigma)$-strain $(\varepsilon)$ curves obtained for freestanding films composed of (a) $100 \mathrm{cP}$ ethyl cellulose (EC) with cross-link densities of 0 (black), 1 (blue), 10 (gray), and 100\% (red) and (b) $300 \mathrm{cP} \mathrm{EC}$ with cross-link densities of 0 (black), 1 (blue), and 10\% (gray). Hexamethylene diisocyanate (HMDI) was used as a cross-linker and all coatings were cured at RT for $120 \mathrm{~h}$ before performing mechanical analysis. (c) Water vapor permeabilities $(P)$ derived from wet-cup experiments for $100 \mathrm{cP}$ EC films as a function of cross-link density.

encapsulation of salt hydrates due to its simplicity and suitability for facile upscaling.

3.4. Effect of Casting Solvent. Four commonly applied solvents, namely, dichloromethane (DCM), ethanol (EtOH), acetone, and dimethylformamide (DMF), were used to investigate the effect of casting medium on the mechanical properties and water vapor permeabilities of the resulting films. EC300 was used for this comparison. The results are summarized in Figure 6. Evidently, the choice of casting solvent has a dramatic influence on the mechanical film properties (Figure 6a). The films cast from DCM were by far the most deformable as manifested by a significantly larger $\varepsilon_{\max }$ as obtained for the films made from DMF-, acetone-, or

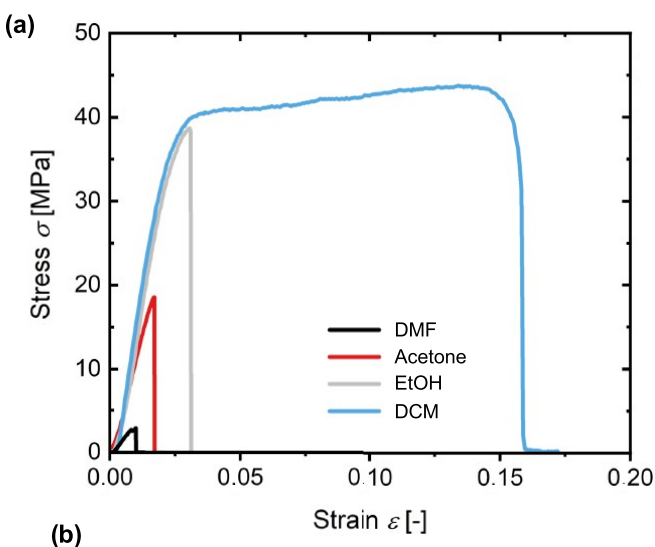

(b)

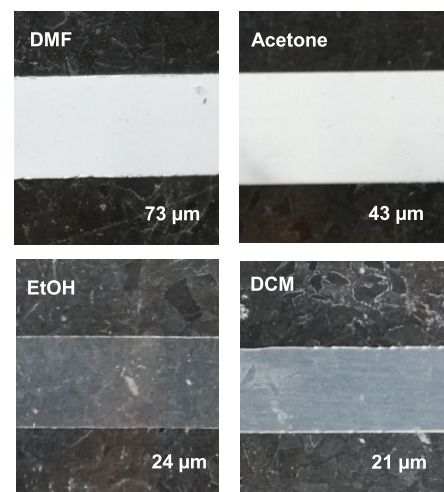

(c)

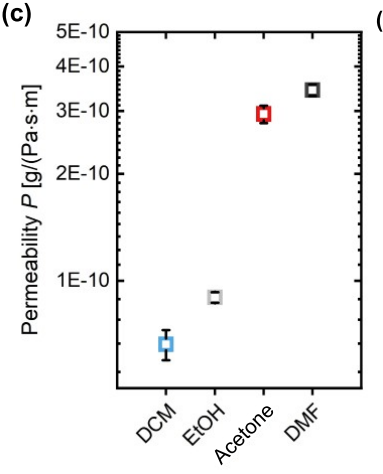

(d) Good solvent Solvated coils

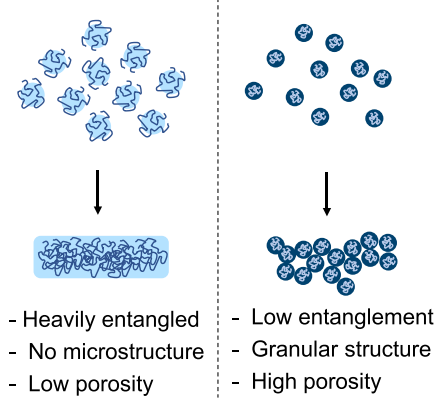

Figure 6. (a) Stress $(\sigma)$-strain $(\varepsilon)$ curves obtained for EC $(300 \mathrm{cP})$ freestanding films cast from DMF (black), acetone (red), EtOH (gray), and DCM (blue). (b) Macroscopic appearances of the coatings cast from the different solvents. The numbers listed in the bottom right corner of the pictures represent the average coating thickness. (c) Water vapor permeability $(P)$ derived from wet-cup experiments for EC films cast from different solvents. (d) Schematic representation of film forming and the resulting film properties using a casting medium, which is a good (left) and poor (right) solvent for the polymers.

ethanol-based polymer solutions. In addition to the failure at low strains, the DMF- and acetone-cast films also have significantly lower strength compared to the films prepared using ethanol or DCM as a solvent. Evidently, DCM should be the solvent of choice when targeting deformable/plastic films. The unfavorable mechanical properties of the acetone- and DMF-based coatings are however complemented with relatively high water vapor permeabilities.

From Figure $6 a-c$, one can conclude that the permeability is inversely proportional to the maximum strain or (yield) strength of the polymer films. Underlying this relation between the mechanical characteristics and permeability is the micro- 
structure of the film. As reported before, the choice of casting solvent has a distinct influence on the microstructure of the solid matrix formed after drying. ${ }^{53}$ These structural variations are directly related to the solvent quality of the casting medium. In media that are able to efficiently solvate the employed polymers (DCM), the chains will be highly swollen (Figure 6d, left). The swelling facilitates the formation of contacts between the polymers upon drying, eventually resulting in heavily entangled polymer materials in which the individual chains are no longer distinguishable. These films have therefore a homogeneous composition up to the molecular length scale. The absence of any structure on larger length scales is manifested by the transparent appearance of these films (Figure 6b, EtOH- and DCM-based coatings). The tight packing of the polymer chains gives a high degree of chain entanglements and hence the capability to accommodate large deformations. Simultaneously, the dense polymer structures lead to low permeabilities for water vapor. In the case of poor solvents (Figure 6b, DMF and acetone), the polymer coils adopt collapsed conformations. During the drying process, these collapsed coils are not able to interact efficiently, leading to a polymer matrix, which has limited entanglements and microstructural features caused by spatial variations in polymer density. Evidence for the presence of this internal structure is provided by the complete white and opaque appearance of the resulting films (Figure 6b, DMF and acetone). The opacity is caused by multiple scattering of visible light between cellulose microstructural elements and air inclusions. ${ }^{54}$ Additionally, the coatings prepared using DMF and acetone were significantly thicker (73 and $43 \mu \mathrm{m}$, respectively) compared to the EtOH and DCM analogues (24 and $21 \mu \mathrm{m}$, respectively) despite equal polymer concentrations in the used casting solutions. This increase in thickness is consistent with the inefficient packing of the polymers in the solid film, as air-filled voids are incorporated. Due to their inhomogeneities and absence of chain entanglements, these films behave rather brittle. Besides acting like mechanical failure sites, the low-polymer-density region in the coating increases the porosity and hence facilitates the transport of water vapor through the polymer matrix.

In summary, the choice of casting solvent is a simple experimental parameter to tune the maximum strains and permeability of the polymer films. Employing good solvents for the polymers results in more deformable/plastic coatings, while films with higher permeabilities can be obtained using solvents that have a low(er) affinity for the polymers.

3.5. Effect of Coating Thickness. In addition to the chemical strategies to alter the properties of the shell material described before, tuning the thickness of the polymer layer is a potential more physical route. Naturally, the total resistance against water vapor transport decreases if the shell becomes thinner, facilitating fast (de)sorption kinetics. From a mechanical property perspective, the force required to deform thinner films is lower. However, as previously established, the polymer coating is not designed to restrict salt expansion by counteracting the solvation-induced pressure (Section 3.1, eq 3). As long as the maximum strain at failure is sufficient to account for the salt swelling, the coating should suffice as a protecting shell.

To find the minimum thickness required to meet the mechanical constraints imposed by the salt swelling, EC300based coatings with average thicknesses of $4,11,15,21$, and 34 $\mu \mathrm{m}$ were fabricated and subjected to tensile testing (Figure 7a). All films were able to withstand approximately the same amount of stress, while the elongation at break differed

(a)

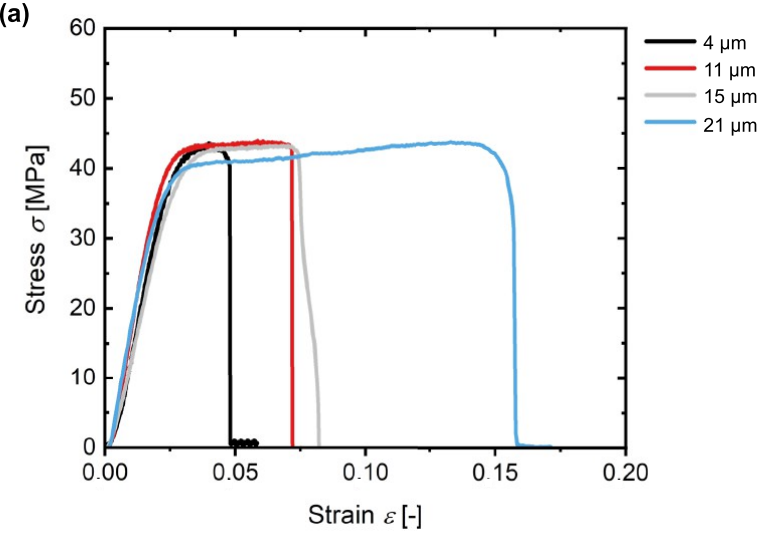

(b)

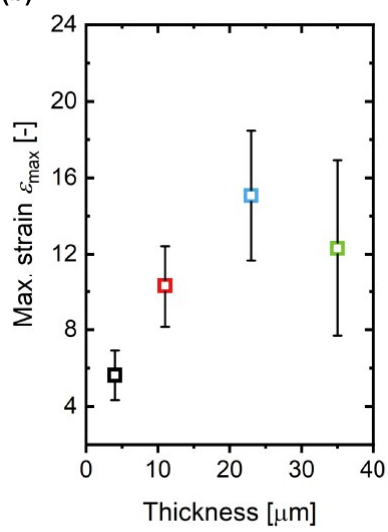

(c)

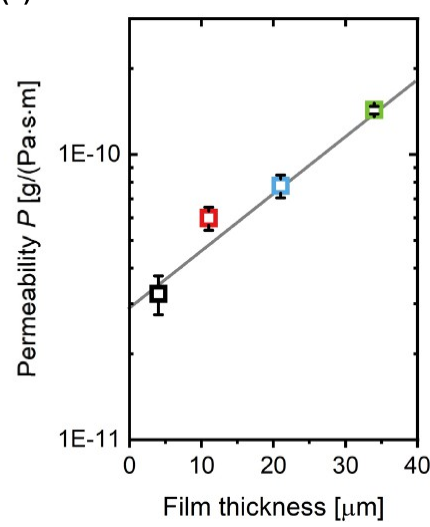

Figure 7. (a) Stress $(\sigma)$-strain $(\varepsilon)$ curves obtained for EC (300 cP) freestanding films with thicknesses of 4 (black), 11 (red), 15 (gray), and $21 \mu \mathrm{m}$ (blue). (b) Maximum achievable strain before failure during tensile testing as a function of coating thickness. (c) Water vapor permeabilities $(P)$ derived from wet-cup experiments for EC coatings $(300 \mathrm{cP})$ as a function of film thickness.

significantly. Upon lowering the film thickness by approximately a factor of 5 , the maximum strain that could be accommodated decreased by a factor of 3 , where a minimum $\varepsilon_{\max }$ of only 0.05 was measured for the $4 \mu \mathrm{m}$ films. As depicted in Figure $7 \mathrm{~b}, \varepsilon_{\max }$ seems to increase with increasing film thickness until the maximum strains plateau at coating thicknesses exceeding $20 \mu \mathrm{m}$. These data suggest that the coatings need an appreciable thickness to accommodate the stresses imposed during the hydration-induced swelling of the salt. The plateauing behavior of $\varepsilon_{\max }$ as a function of the coating thickness can be explained by the transition from surface to bulk dominated samples. Due to the fabrication process, it is probable that the films possess a surface layer with a different polymer morphology as the bulk of the material. This layer is the result of faster drying of the wet coating at the air-solution interface. After the formation of a dried surface polymer layer, the rate of solvent evaporation from the remainder of the wet coating is decreased, giving more time to the polymer chains to find (more) optimal configurations. Since the mechanical properties of polymer films highly depend on their internal structure, the bulk and surface layers might have different $\varepsilon_{\max }$ values. In agreement with this reasoning, the existence of surface layers with vastly different 

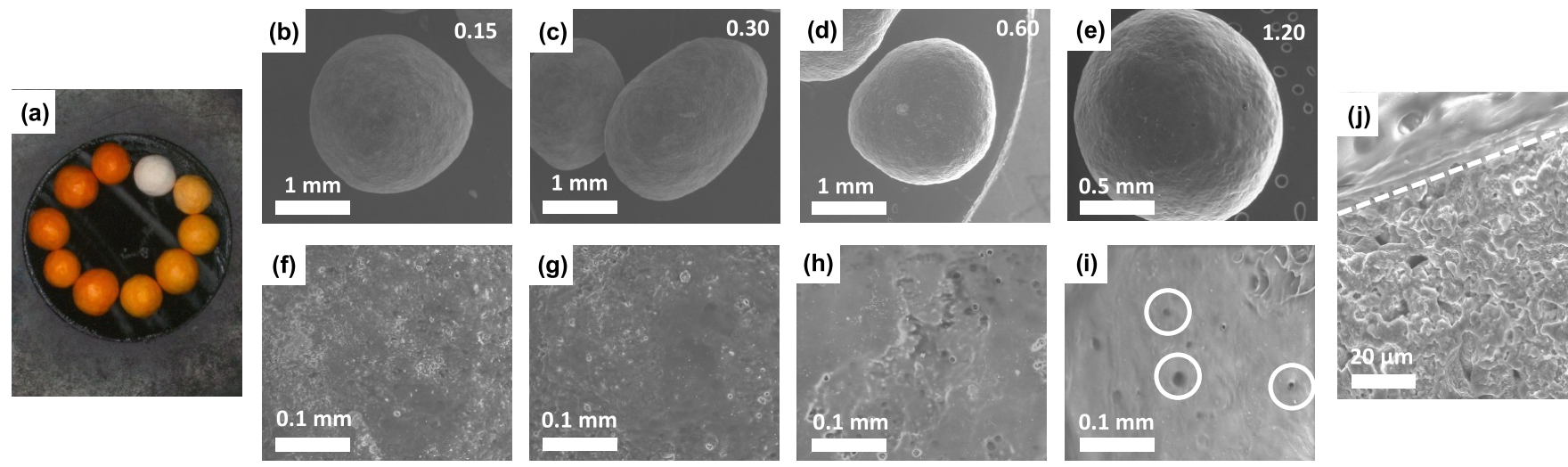

Figure 8. (a) Macroscopic appearance of zeolite grains coated with EC100 (colored with Disperse Orange 3, DO3) as a function of time. The coating time, and hence the deposited quantity of polymer increases from the top white grain clockwise to the intensely colored beads. Scanning electron microscopy (SEM) images of $\mathrm{K}_{2} \mathrm{CO}_{3}$ grains during a coating process with EC100 containing (b, f) $0.15,(c, g) 0.30,(d, h) 0.60$, and (e, i) $1.20 \mathrm{wt} \%$ polymer. The white circles in (i) highlight surface defects of the polymer coating. (j) Cross section of the grain depicted in (e). The white dotted line indicates an estimation of the salt-polymer interface.

physical properties in polymeric coatings was previously reported. ${ }^{55}$

Additional evidence for the presence of such surface layer is provided by permeability measurements. Figure $7 \mathrm{c}$ shows a plot of permeability versus layer thickness. The plotted permeabilities are normalized to the layer thickness and since the films are composed of identical polymers and prepared via identical procedures, equal water vapor permeabilities were expected. On the contrary, the permeability increases with increasing layer thickness. ${ }^{45}$ This observation could be explained by the presence of a surface layer that is less permeable for water vapor. Coatings are known to have varying structural properties throughout their thickness caused by differences in drying rate. ${ }^{58-58}$ For thin coatings, this layer dominates, while for the thicker coating, the bulk of the film dictates the permeability measurements. From these results, one could conclude that thicker coatings favor water vapor transport. However, when consulting eq 2 , the water vapor flux $(\mathrm{d} Q / \mathrm{d} t)$ across the coating is also inversely proportional to the coating thickness $(d)$. Increasing $d$ imposes a longer diffusive pathway, which slows down vapor transport. Therefore, the trade-off between thickness and associated permeability needs to be considered when targeting certain heat storage capabilities.

3.6. Coating of TCM and Zeolite Grains. Based on the findings in terms of mechanical properties and water vapor permeabilities for the macroscopic freestanding films, granular matter was encapsulated in a polymer shell by means of fluidized bed coating. Besides using granular $\mathrm{K}_{2} \mathrm{CO}_{3}$ (1-2 $\mathrm{mm})$, zeolite grains (8-12 mesh) were used as a model system. In contrast to the salt hydrates targeted for the heat battery applications, zeolite grains do not undergo volumetric changes upon (de)hydration, making it an ideal system to study the effect of an encapsulating polymer layer on the water vapor transport kinetics. We must note that the zeolites, despite their high tendency to adsorb water from its surroundings into its nanoporous internal structure, are not suitable for the foreseen application as the material has too high regeneration temperatures $\left(>150{ }^{\circ} \mathrm{C}\right)$ and too low energy storage densities $\left(<0.4 \mathrm{GJ} / \mathrm{m}^{3}\right) .{ }^{59}$ Fluidized bed coating was selected as it can be easily scaled up to industrially relevant quantities. A detailed description of the process can be found in Section 2.5. In short, a bed of granular material is fluidized by applying a continuous stream of air under the particle bed. Once a stable fluid bed is formed, polymer solution is sprayed onto the moving granules via an atomizing fluid nozzle. Since the bed is kept at an elevated temperature, the solvent evaporated quickly as the polymer is deposited on the granules. Optimization of the operating conditions in terms of fluidization air flow, polymer addition speed, viscosity of the polymer solution, bed temperature, and atomization nozzle dimensions leads to the formation of homogeneous coatings and a controlled growth of the shell. Illustrative results of the fluidized bed coating process are depicted in Figure 8, where examples for zeolite and $\mathrm{K}_{2} \mathrm{CO}_{3}$ grains are shown. We would like to emphasize that this coating strategy can be easily extended to any arbitrary material or granule shape, as long as the particles can be efficiently fluidized. The polymers used as coating material can be varied. Both water- and solvent-based polymer solutions can be employed.

To visually monitor the progression of the coating process, a dye (Disperse Orange 3, DO3 for EC100-based solutions and methylene blue, MB for PVA-based solutions) was added to the polymer solution, resulting in a gradual increase in the intensity of the color of the coated granular material. The time evolution of the macroscopic appearance of the EC100 coated zeolite grains is depicted in Figure 8a. The orange color was evenly distributed over the granule surface, indicative of controlled and homogeneous growth of the polymer shell. Furthermore, fluidization did not lead to mechanical degradation of the particles as both size and shape were retained throughout the coating process. The macroscopic assessment of the coating quality was corroborated with scanning electron microscopy (SEM) analysis of the coated $\mathrm{K}_{2} \mathrm{CO}_{3}$ beads as a function of the coating time (Figure $8 \mathrm{~b}-\mathrm{i}$ ). The weight percentage of added polymer was calculated based on the total mass of the granules and the mass of polymer solution added with a known concentration after a certain coating time. After addition of $0.15-0.3$ wt \% EC100, the irregular surface structure of the salt grains remains visible on the complete grain. Increasing the weight percentage of polymer resulted in salt grains that were nearly covered by a smooth layer of polymer, while small uncoated domains could still be observed. Supplying an excess of $1 \mathrm{wt} \%$ of polymer to the grains led to full encapsulation. Despite a low concentration of micron-sized surface defects (Figure 8i, 

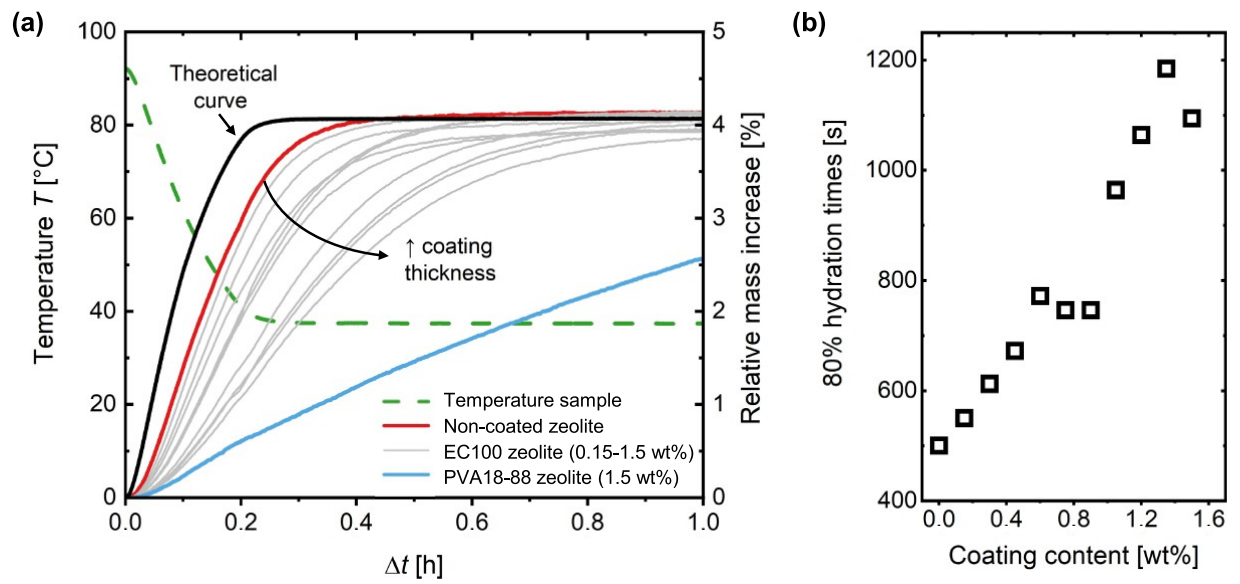

Figure 9. (a) Hydration kinetics of zeolite coated with different weight percentages (wt \%) of EC100. The theoretical curve is constructed based on the assumption that the grain is in equilibrium with the applied temperature and water vapor pressure. As a reference, the hydration kinetics of zeolites coated with $1.5 \mathrm{wt} \%$ poly(vinyl alcohol) (PVA18-88) are included (blue curve). Measurements were performed at a water vapor pressure of 12 mbar. (b) Average mass percentage of coating material per zeolite grain versus the time required to reach a mass that corresponds to $80 \%$ of the fully hydrated state.

white circles), a homogeneously smooth polymer surface was obtained. Based on the added quantity of polymer, the minimum layer thickness to achieve full encapsulation is on the order of 5-10 $\mu \mathrm{m}$, which is comparable to the thickness of the freestanding films used for mechanical and wet-cup testing. SEM analysis of the cross section of a coated $\mathrm{K}_{2} \mathrm{CO}_{3}$ grain (1.2 wt $\%$ polymer, Figure $8 \mathbf{j}$ ) confirmed this estimation. Probing the coating thickness on multiple locations on comparable grains by optical microscopy analysis (Section S10, Supporting Information) yielded a consistent value of $10.5 \pm 1.4 \mu \mathrm{m}$. The relatively small variation in thickness highlights the coating's uniformity. Naturally, being able to grow such thin shells is advantageous in terms of water vapor permeability. Increasing the shell thickness to, for example, improve the mechanical resilience and ductility can be simply achieved by prolonging the coating time.

As fluidized bed coating is fundamentally different from the procedure employed to fabricate the previously investigated freestanding films, an initial study toward measuring the water vapor permeability of the coated grains was performed to verify the validity of using the macroscopic coatings as a representative model for the shell materials. To this end, the hydration kinetics were measured for zeolites coated with 100 cP EC using thermogravimetric analysis (TGA), as described in Section 2.6. The results of these TGA measurements are summarized in Figure 9, where the hydration curves of the uncoated zeolite (red), 10 zeolite samples with varying coating thickness (gray), and the theoretical hydration curve (black) are plotted. The theoretical curve was calculated based on the measured environmental temperature, the applied water vapor pressure, and the adsorption curves of the employed zeolite. ${ }^{59}$ For this calculation, equilibrium between the adsorption and supplied water was assumed, i.e., an infinitely fast system.

From these TGA data, three key observations can be extracted: (i) the maximum mass increase at the end of the hydration experiment is equal for all samples and similar to the theoretical maximum, indicative of the fact that full hydration at the given temperature and water vapor pressure can be reached regardless of the presence of the EC coating. (ii) The uncoated zeolite sample hydrates slower than theoretically predicted based on the applied conditions. This indicates that the sample is not in equilibrium during the entire experiment, which will result in an underestimation of the permeability of the coating. (iii) The coating decreases the water vapor uptake rates of the zeolites. By increasing the thickness of the EC layer, longer hydration times are required to reach the fully hydrated state. The relation between the coating thickness and the water vapor uptake rate is emphasized in Figure $9 \mathrm{~b}$ where the average mass percentage of coating material per zeolite grain is plotted versus the time required to reach a mass that corresponds to $80 \%$ of the fully hydrated state.

Based on the TGA experiments, the water vapor permeability of the coatings was approximated (Figure 10). This estimation is based on the following assumptions: (i) the polymer coating forms a homogeneous layer without porosity in the form of microscopic cracks or holes and has a uniform thickness that can be calculated based on the applied coating

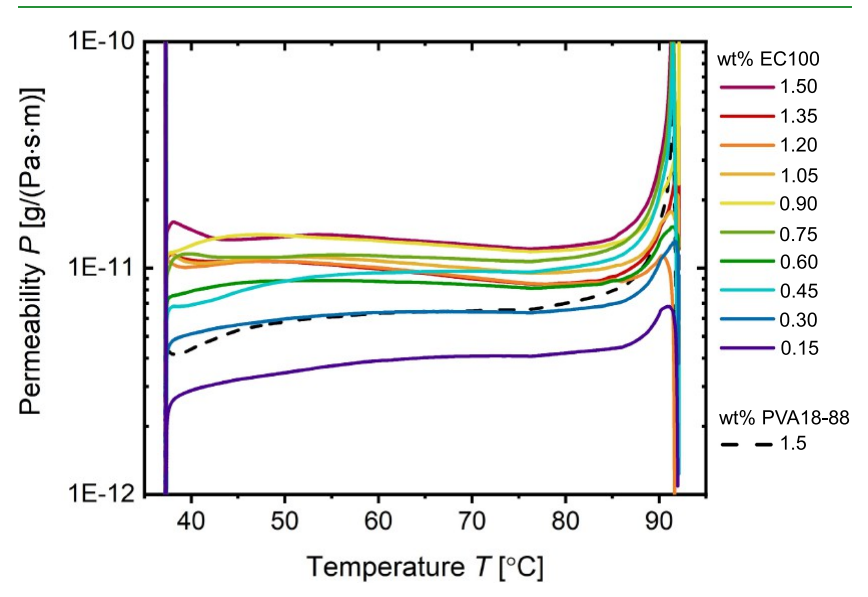

Figure 10. Water vapor permeability $(P)$ of zeolite grains coated with $100 \mathrm{cP}$ ethyl cellulose (EC100) films as a function of the weight percentage of supplied polymer (range of coating: $0.15-1.5$ wt $\%$ with increments of $0.15 \mathrm{wt} \%)$. As a reference, zeolite coated with $1.5 \mathrm{wt} \%$ poly(vinyl alcohol) (PVA18-88) was included (black dashed curve). The samples with the two thinnest coatings have a significantly different permeability compared to the others. The variations of permeabilities of samples containing $0.45-1.5 \mathrm{wt} \%$ of polymer are within the error of the calculation method. 

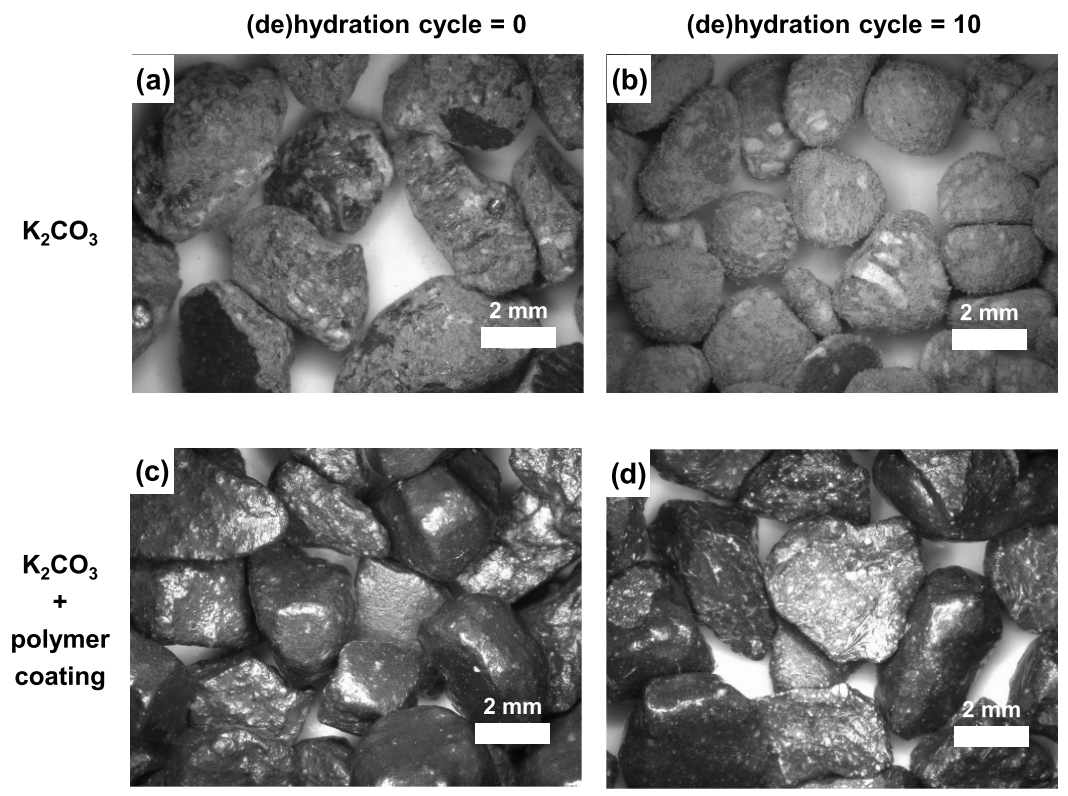

Figure 11. Macroscopic appearance of (a, b) uncoated and (c, d) HPC-coated $\mathrm{K}_{2} \mathrm{CO}_{3}$-based grains. The images in (a) and (c) were taken on fresh grains, while those in (c) and (d) show the particles after 10 consecutive (de)hydration cycles. Test conditions per cycle: $30{ }^{\circ} \mathrm{C}$ at 14 mbar hydration for $20 \mathrm{~h} ; 120^{\circ} \mathrm{C}$ at 14 mbar dehydration for $5 \mathrm{~h}$.

mass and the bulk density of EC; (ii) the water activity inside the zeolite can be calculated based on the $p T$ curves assuming that the loading in the zeolite grain is homogeneously distributed over the entire experiment and the temperature of the sample is equal to the environmental temperature imposed by the oven. As a reference, the hydration kinetics of zeolite coated with PVA18-88 were measured as well. In agreement with the wet-cup experiments on the macroscopic freestanding films (Figure 2), the water vapor permeability is significantly lower for coatings composed of this polymer compared to their EC100-based counterparts.

With exception of the two thinnest coatings, the water vapor permeability of all of the coatings was found to be on the order of $10^{-11} \mathrm{~g} /(\mathrm{Pa} \cdot \mathrm{s} \cdot \mathrm{m})$. This discrepancy is caused by the fact that the calculation is rather sensitive to the exact coating thickness. Errors made in the estimation of this thickness are probably largest for the thin films. Considering that the coating applied onto the zeolites is on the order of a few micrometers (Figure $8 j$ ), the permeabilities are in good agreement with the values for the freestanding films (Figure 7c). Surprisingly, the vastly different fabrication routes and the appearance of (sub)micrometer defects in the coating applied by fluidized bed coating (Figure $8 \mathrm{i}$, white circles), do not result in a dramatic mismatch in water vapor permeability. In contrast to the freestanding films, where the permeability increases with increasing film thickness (Figure 7c), fairly constant permeabilities were found from zeolites containing $0.5-1.5 \mathrm{wt} \%$ polymer. This difference in behavior can be explained when considering the formation mechanism of both types of polymer coating. In contrast to the freestanding films which are made in a single step, the polymer films on the zeolite grains are introduced stepwise by random encounters of the particles with supplied polymer solution during the fluidized bed coating. In addition, since the coating process is performed at elevated temperatures, evaporation of the solvent (DCM) is fast. This implies that the polymers, once deposited onto the surface of the zeolite grains, have limited opportunity to rearrange and form dense or intertwined polymeric films.
Consequently, the final polymer shell will resemble a stack of thin coatings. The overall permeability of this shell will therefore be limited by the permeability of these individual thin layers and will not change as a function of coating time.

From this preliminary evaluation, it appears that the wet-cup experiments provide a qualitative indicator for the permeability of polymer coatings applied by fluidized bed processes. Despite that the absolute numbers for the permeability are different, the same trends as observed for the freestanding films when comparing different coating chemistries. The permeability of PVA-based coatings derived from wet-cup and TGA measurements are both significantly lower compared to the values obtained for EC100 films. This implies that the wet-cup results (Figure 2) can still be used to internally compare and screen for applicability of different polymers as coating material for salt hydrate grains.

Finally, the effect of the coating on the stability of $\mathrm{K}_{2} \mathrm{CO}_{3}$ based grains during repetitive (de)hydration was investigated. Uncoated and coated grains were subjected to 10 (de)hydration cycles. For this particular experiment, salt granules containing a carbon-based binder were used, causing their dark appearance. HPC was selected as the polymer of choice. The macroscopic appearance of the grains before and after this procedure is shown in Figure 11 (a large overview picture of the salt grains can be found in Section S11, Supporting Information). For the uncoated salt, the (de)hydration cycles lead to a morphological change of the grains, which develop a fibrous surface structure (Figure 10b). Together with an increased embrittlement, this behavior is detrimental for the heat storage capabilities when extrapolated to large beds of grains and significantly more (de)hydration cycles. In contrast, the coated granules (Figure 10c,d) remain smooth and intact after (de)hydration. No rupture of the coating was observed, proving that the ductility as predicted by the tensile tests is sufficient to accommodate the hydration-induced swelling of the salt. These preliminary results are a promising starting point for testing these coated grains on a larger scale and through more (de)hydration cycles. 


\section{CONCLUSIONS}

The applicability of a variety of cellulose and poly(vinyl alcohol) derivates as coating materials for the stabilization of salt hydrates for heat storage applications was investigated. A systematic study on the maximum degree of deformability and the water vapor permeability of films composed from these polymers was performed. Coating deformation is required to safeguard that the coating does not rupture during hydrationinduced swelling of the salt. A high water permeability is desired to maximize the power of the resulting heat battery by limiting the resistance for water vapor transport from and to the active salt hydrate core. To this end, well-defined freestanding films were prepared, which were subjected to tensile testing and wet-cup experiments. Ethyl and hydroxypropyl cellulose proved to be promising candidates combining ductility with sufficient water vapor permeabilities. Detailed investigation on the effects of casting solvent, molecular weight, degree of cross-linking, and the coating thickness revealed that the mechanical demands imposed by the hydration-induced swelling of the salts can most probably be met by selecting high-molecular-weight polymers, casting from a good solvent, or fabricate coatings with a moderate cross-link density. The typical water vapor permeabilities $\left(\sim 10^{-11} \mathrm{~g} /(\mathrm{Pa} \cdot \mathrm{s} \cdot \mathrm{m})\right)$ of the fabricated coatings proved to be compatible with low-power residential applications of a TCMbased heat battery. Incorporation of (micro)porosity via inclusion of sacrificial porogens ${ }^{60,61}$ or the use of phaseseparating polymer blends/solutions ${ }^{34,62,63}$ are among potential strategies to increase the porosity of the coatings and are recommended for follow-up studies. For the studied films, no significant changes were found in the permeability as a function of the molecular weight or cross-link density. Casting films from poor solvents did increase the permeability but resulted in coatings that were extremely brittle and therefore not suitable. The coating thickness has a distinct influence on the permeability. The permeability increases with increasing coating thickness, a behavior that can be explained by the presence of a dense rather impermeable surface layer of the coatings as a consequence of the fabrication procedure for the freestanding films.

Finally, the results for the freestanding films were translated to coatings applied to granular material (zeolite and $\mathrm{K}_{2} \mathrm{CO}_{3}$ ) via fluidized bed coating. Performing detailed TGA measurements on these coated granules resulted in water vapor permeabilities that agreed with the values obtained for thin freestanding films. Although a quantitative comparison between cast and fluidized-bed-coated films is precarious due to their completely different fabrication methods, the same trends in permeability were found when comparing films of different chemistry. Furthermore, subjecting the coated $\mathrm{K}_{2} \mathrm{CO}_{3}$ grains to 10 consecutive (de)hydration cycles showed that the polymer layer remained intact and greatly improved the mechanical resilience of the granules compared to their noncoated counterparts. Therefore, the study on the films provides a reasonable and straightforward experimental test case to pre-select promising materials as coating materials for salt hydrates. Future studies will focus on assessing the chemical stability over prolonged cycling and performance in real-life heat battery environments.

\section{ASSOCIATED CONTENT}

\section{(s) Supporting Information}

The Supporting Information is available free of charge at https://pubs.acs.org/doi/10.1021/acsapm.0c01186.

Comparison energy densities of pure, granular, and stabilized $\mathrm{K}_{2} \mathrm{CO}_{3}$; required water vapor permeability as a function of grain size; overview of the setup used for freestanding film preparation; calculation of cross-link densities of the ethyl cellulose films; experimental settings of the fluidized bed coating of zeolite and $\mathrm{K}_{2} \mathrm{CO}_{3}$ grains; overview of the tensile testing setup; water vapor permeability $(P)$ of polymeric films as a function of time; estimation of tensile pulling rates based on the kinetics of hydration-induced salt expansion; infrared (IR) spectroscopy of cross-linking EC100 with 1 and 100\% HMDI; thickness determination of coating on $\mathrm{K}_{2} \mathrm{CO}_{3}$ grains using optical microscopy; and overview picture of (un) coated $\mathrm{K}_{2} \mathrm{CO}_{3}$ grains before and after 10 (de)hydration cycles (PDF)

\section{AUTHOR INFORMATION}

\section{Corresponding Author}

Bas G. P. van Ravensteijn - The Netherlands Organization

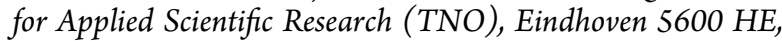
The Netherlands; ㅇo orcid.org/0000-0001-9024-3927; Email: b.g.p.v.ravensteijn@tue.nl, hartmut.fischer@tno.nl

\section{Authors}

Pim A. J. Donkers - The Netherlands Organization for Applied Scientific Research (TNO), Eindhoven 5600 HE, The Netherlands

Rick C. Ruliaman - Department of Applied Physics, Eindhoven University of Technology, Eindhoven 5600 MB, The Netherlands

Jacco Eversdijk - The Netherlands Organization for Applied Scientific Research (TNO), Eindhoven $5600 \mathrm{HE}$, The Netherlands

Hartmut R. Fischer - The Netherlands Organization for Applied Scientific Research (TNO), Eindhoven 5600 HE, The Netherlands; o orcid.org/0000-0001-9724-4922

Henk P. Huinink - Department of Applied Physics, Eindhoven University of Technology, Eindhoven 5600 MB, The Netherlands

Olaf C. G. Adan - The Netherlands Organization for Applied Scientific Research (TNO), Eindhoven $5600 \mathrm{HE}$, The Netherlands; Department of Applied Physics, Eindhoven University of Technology, Eindhoven 5600 MB, The Netherlands

Complete contact information is available at:

https://pubs.acs.org/10.1021/acsapm.0c01186

\section{Author Contributions}

${ }^{\S}$ Department of Chemical Engineering and Chemistry, Institute for Complex Molecular Systems, Eindhoven University of Technology, P.O. Box 513, Eindhoven 5600 MB, The Netherlands.

\section{Notes}

The authors declare no competing financial interest.

\section{ACKNOWLEDGMENTS}

Henk van Bracht (TNO) is thanked for guidance during fabrication and analysis of the freestanding polymer films. The 
authors thank the Top Consortia for Knowledge and Innovation (TKI) Urban Energy for funding (Project: Characterization of Materials and Systems for Thermal Energy Storage (COMSTES), Project number: 1407204). They also thank Henry van der Meer (Caldic B.V.) for supplying the composite materials.

\section{REFERENCES}

(1) Employment Effects of Selected Scenarios from the Energy Roadmap 2050, Final Report for the European Commission (DG Energy); Cambridge Econometrics, 2013.

(2) Eurostat. Energy Consumption in Households; Eurostat, 2018.

(3) Frazzica, A.; Cabeza, L. F., Eds. Recent Advancements in Materials and Systems for Thermal Energy Storage-An Introduction to Experimental Characterization Methods; Springer International Publishing, 2019.

(4) Shchukina, E. M.; Graham, M.; Zheng, Z.; Shchukin, D. G. Nanoencapsulation of Phase Change Materials for Advanced Thermal Energy Storage Systems. Chem. Soc. Rev. 2018, 47, 4156-4175.

(5) Donkers, P. A. J.; Sögütoglu, L. C.; Huinink, H. P.; Fischer, H. R.; Adan, O. C. G. A Review of Salt Hydrates for Seasonal Heat Storage in Domestic Applications. Appl. Energy 2017, 199, 45-68.

(6) Xie, N.; Huang, Z.; Luo, Z.; Gao, X.; Fang, Y.; Zhang, Z. Inorganic Salt Hydrate for Thermal Energy Storage. Appl. Sci. 2017, 7, No. 1317.

(7) Mao, J.; Hou, P.; Liu, R.; Chen, F.; Dong, X. Preparation and Thermal Properties of SAT-CMC-DSP/EG Composite as Phase Change Material. Appl. Therm. Eng. 2017, 119, 585-592.

(8) Duan, Z. J.; Zhang, H. Z.; Sun, L. X.; Cao, Z.; Xu, F.; Zou, Y. J.; Chu, H. L.; Qiu, S. J.; Xiang, C. L.; Zhou, H. Y. $\mathrm{CaCl}_{2} \cdot 6 \mathrm{H}_{2} \mathrm{O}$ / Expanded Graphite Composite as Form-Stable Phase Change Materials for Thermal Energy Storage. J. Therm. Anal. Calorim. 2014, 115, 111-117.

(9) Xu, B.; Li, Z. Paraffin/Diatomite Composite Phase Change Material Incorporated Cement-Based Composite for Thermal Energy Storage. Appl. Energy 2013, 105, 229-237.

(10) Zhang, Y. N.; Wang, R. Z.; Zhao, Y. J.; Li, T. X.; Riffat, S. B.; Wajid, N. M. Development and Thermochemical Characterizations of Vermiculite/ $\mathrm{SrBr}_{2}$ Composite Sorbents for Low-Temperature Heat Storage. Energy 2016, 115, 120-128.

(11) Permyakova, A.; Wang, S.; Courbon, E.; Nouar, F.; Heymans, N.; D’Ans, P.; Barrier, N.; Billemont, P.; De Weireld, G.; Steunou, N.; Frère, M.; Serre, C. Design of Salt-Metal Organic Framework Composites for Seasonal Heat Storage Applications. J. Mater. Chem. A 2017, 5, 12889-12898.

(12) Courbon, E.; D’Ans, P.; Permyakova, A.; Skrylnyk, O.; Steunou, N.; Degrez, M.; Frère, M. Further Improvement of the Synthesis of Silica Gel and $\mathrm{CaCl}_{2}$ Composites: Enhancement of Energy Storage Density and Stability over Cycles for Solar Heat Storage Coupled with Space Heating Applications. Sol. Energy 2017, 157, 532-541.

(13) Yang, K.; Shi, Y.; Wu, M.; Wang, W.; Jin, Y.; Li, R.; Shahzad, M. W.; Ng, K. C.; Wang, P. Hollow Spherical $\mathrm{SiO}_{2}$ Micro-Container Encapsulation of $\mathrm{LiCl}$ for High-Performance Simultaneous Heat Reallocation and Seawater Desalination. J. Mater. Chem. A 2020, 8, 1887-1895.

(14) Shkatulov, A.; Joosten, R.; Fischer, H.; Huinink, H. Core-Shell Encapsulation of Salt Hydrates into Mesoporous Silica Shells for Thermochemical Energy Storage. ACS Appl. Energy Mater. 2020, 3, 6860-6869.

(15) Shin, H. K.; Park, M.; Kim, H. Y.; Park, S. J. Thermal Property and Latent Heat Energy Storage Behavior of Sodium Acetate Trihydrate Composites Containing Expanded Graphite and Carboxymethyl Cellulose for Phase Change Materials. Appl. Therm. Eng. 2015, 75, 978-983.

(16) Li, Y.; Yu, S.; Chen, P.; Rojas, R.; Hajian, A.; Berglund, L. Cellulose Nanofibers Enable Paraffin Encapsulation and the Formation of Stable Thermal Regulation Nanocomposites. Nano Energy 2017, 34, 541-548.
(17) Ryu, H. W.; Woo, S. W.; Shin, B. C.; Kim, S. D. Prevention of Supercooling and Stabilization of Inorganic Salt Hydrates as Latent Heat Storage Materials. Sol. Energy Mater. Sol. Cells 1992, 27, 161172.

(18) Nonnen, T.; Preißler, H.; Kött, S.; Beckert, S.; Gläser, R. Salt Inclusion and Deliquescence in Salt/Zeolite $\mathrm{X}$ Composites for Thermochemical Heat Storage. Microporous Mesoporous Mater. 2020, 303, No. 110239.

(19) Nonnen, T.; Beckert, S.; Gleichmann, K.; Brandt, A.; Unger, B.; Kerskes, H.; Mette, B.; Bonk, S.; Badenhop, T.; Salg, F.; Gläser, R. A Thermochemical Long-Term Heat Storage System Based on a Salt/ Zeolite Composite. Chem. Eng. Technol. 2016, 39, 2427-2434.

(20) Xu, J. X.; Li, T. X.; Chao, J. W.; Yan, T. S.; Wang, R. Z. High Energy-Density Multi-Form Thermochemical Energy Storage Based on Multi-Step Sorption Processes. Energy 2019, 185, 1131-1142.

(21) Ur Rehman, A.; Maosheng, Z.; Hayat, A. Water Sorption Studies on $\mathrm{ZnSO}_{4}$-Zeolite Composite as Potential Thermochemical Heat Storage Materials. Int. J. Energy Res. 2020, 44, 269-281.

(22) Salunkhe, P. B.; Shembekar, P. S. A Review on Effect of Phase Change Material Encapsulation on the Thermal Performance of a System. Renewable Sustainable Energy Rev. 2012, 16, 5603-5616.

(23) Milián, Y. E.; Gutiérrez, A.; Grágeda, M.; Ushak, S. A Review on Encapsulation Techniques for Inorganic Phase Change Materials and the Influence on Their Thermophysical Properties. Renewable Sustainable Energy Rev. 2017, 73, 983-999.

(24) Jamekhorshid, A.; Sadrameli, S. M.; Farid, M. A Review of Microencapsulation Methods of Phase Change Materials (PCMs) as a Thermal Energy Storage (TES) Medium. Renewable Sustainable Energy Rev. 2014, 31, 531-542.

(25) Liu, C.; Wang, C.; Li, Y.; Rao, Z. Preparation and Characterization of Sodium Thiosulfate Pentahydrate/Silica Microencapsulated Phase Change Material for Thermal Energy Storage. RSC Adv. 2017, 7, 7238-7249.

(26) Graham, M.; Shchukina, E.; De Castro, P. F.; Shchukin, D. Nanocapsules Containing Salt Hydrate Phase Change Materials for Thermal Energy Storage. J. Mater. Chem. A 2016, 4, 16906-16912.

(27) Wang, T.; Huang, J.; Zhu, P.; Xiao, J. Fabrication and Characterization of Micro-Encapsulated Sodium Phosphate Dodecahydrate with Different Crosslinked Polymer Shells. Colloid Polym. Sci. 2013, 291, 2463-2468.

(28) Borreguero, A. M.; Valverde, J. L.; Rodríguez, J. F.; Barber, A. H.; Cubillo, J. J.; Carmona, M. Synthesis and Characterization of Microcapsules Containing RubithermRT27 Obtained by Spray Drying. Chem. Eng. J. 2011, 166, 384-390.

(29) Heßbrügge, B. J.; Vaidya, A. M. Preparation and Characterization of Salt Hydrates Encapsulated in Polyamide Membranes. J. Membr. Sci. 1997, 128, 175-182.

(30) Beving, M. A. J. M.; Frijns, A. J. H.; Rindt, C. C. M.; Smeulders, D. M. J. Effect of Cycle-Induced Crack Formation on the Hydration Behaviour of $\mathrm{K}_{2} \mathrm{CO}_{3}$ Particles: Experiments and Modelling. Thermochim. Acta 2020, 692, No. 178752.

(31) EU H2020 CREATE Project. EeB-06-2015, 2020.

(32) Houben, J.; Sögütoglu, L.; Donkers, P.; Huinink, H.; Adan, O. $\mathrm{K}_{2} \mathrm{CO}_{3}$ in Closed Heat Storage Systems. Renewable Energy 2020, 166, $35-44$.

(33) Marin, E.; Rojas, J.; Ciro, Y. A Review of Polyvinyl Alcohol Derivatives: Promising Materials for Pharmaceutical and Biomedical Applications. Afr. J. Pharm. Pharmacol. 2014, 8, 674-684.

(34) Narisawa, S.; Yoshino, H.; Hirakawa, Y.; Noda, K. PorosityControlled Ethylcellulose Film Coating. I. Formation of Porous Ethylcellulose Film in the Casting Process and Factors Affecting FilmDensity. Chem. Pharm. Bull. 1993, 41, 329-334.

(35) Zhang, G.; Schwartz, J. B.; Schnaare, R. L. Bead Coating. I. Change in Release Kinetics (and Mechanism) Due to Coating Levels. Pharm. Res. 1991, 8, 331-335.

(36) Siepmann, F.; Hoffmann, A.; Leclercq, B.; Carlin, B.; Siepmann, J. How to Adjust Desired Drug Release Patterns from EthylcelluloseCoated Dosage Forms. J. Controlled Release 2007, 119, 182-189. 
(37) Sangaj, N. S.; Malshe, V. C. Permeability of Polymers in Protective Organic Coatings. Prog. Org. Coat. 2004, 50, 28-39.

(38) Kazlauske, J.; Gårdebjer, S.; Almer, S.; Larsson, A. The Importance of the Molecular Weight of Ethyl Cellulose on the Properties of Aqueous-Based Controlled Release Coatings. Int. J. Pharm. 2017, 519, 157-164.

(39) Andersson, H.; Hjärtstam, J.; Stading, M.; Von Corswant, C.; Larsson, A. Effects of Molecular Weight on Permeability and Microstructure of Mixed Ethyl-Hydroxypropyl-Cellulose Films. Eur. J. Pharm. Sci. 2013, 48, 240-248.

(40) Steiger, M. Crystal Growth in Porous Materials - I: The Crystallization Pressure of Large Crystals. J. Cryst. Growth 2005, 282, 455-469.

(41) Wellons, J. D.; Stannett, V. Permeation, Sorption, and Diffusion of Water in Ethyl Cellulose. J. Polym. Sci., Part A-1: Polym. Chem. 1966, 4, 593-602.

(42) Park, H. J.; Chinnan, M. S. Gas and Water Vapor Barrier Properties of Edible Films from Protein and Cellulosic Materials. J. Food Eng. 1995, 25, 497-507.

(43) Debeaufort, F.; Voilley, A.; Meares, P. Water Vapor Permeability and Diffusivity through Methylcellulose Edible Films. J. Membr. Sci. 1994, 91, 125-133.

(44) Doty, P. M.; Aiken, W. H.; Mark, H. Water Vapor Permeability of Organic Films. Ind. Eng. Chem., Anal. Ed. 1944, 16, 686-690.

(45) Xianda, Y.; Anlai, W.; Suqin, C. Water-Vapor Permeability of Polyvinyl Alcohol Films. Desalination 1987, 62, 293-297.

(46) Ethocel: Ethylcellulose Polymers Technical Handbook; DOW Chemical, 2005

(47) Inukai, K.; Takahashi, Y.; Murakami, S.; Ri, K.; Shin, W. Molecular Weight Dependence of Ethyl Cellulose Adsorption Behavior on (La, $\mathrm{Sr})(\mathrm{Ti}, \mathrm{Fe}) \mathrm{O}_{3}-\delta$ Particles in Organic Solvent Pastes and Their Printing Properties. Ceram. Int. 2014, 40, 12319-12325.

(48) Ogita, T.; Yamamoto, R.; Suzuki, N.; Ozaki, F.; Matsuo, M. Molecular-Weight Dependence of Morphology and Mechanical Properties of Ultrahigh-Molecular-Weight Polyethylene Gel Films. Polymer 1991, 32, 822-834.

(49) Mandelkern, L. The Crystallization of Flexible Polymer Molecules. Chem. Rev. 1956, 56, 903-958.

(50) Qiu, X.; Tao, S.; Ren, X.; Hu, S. Modified Cellulose Films with Controlled Permeatability and Biodegradability by Crosslinking with Toluene Diisocyanate under Homogeneous Conditions. Carbohydr. Polym. 2012, 88, 1272-1280.

(51) Mitchell, G. E.; Wilson, L. R.; Dineen, M. T.; Urquhart, S. G.; Hayes, F.; Rightor, E. G.; Hitchcock, A. P.; Ade, H. Quantitative Characterization of Microscopic Variations in the Cross-Link Density of Gels. Macromolecules 2002, 35, 1336-1341.

(52) Benavente, R.; Mijangos, C.; Perena, J. M.; Krumova, M.; López, D. Effect of Crosslinking on the Mechanical and Thermal Properties of Poly (Vinyl Alcohol). Polymer 2000, 41, 9265-9272.

(53) Briscoe, B. J.; Akram, A.; Adams, M. J.; Johnson, S. A.; Gorman, D. M. The Influence of Solvent Quality on the Mechanical Properties of Thin Cast Isotactic Poly(Methyl Methacrylate) Coatings. J. Mater. Sci. 2002, 37, 4929-4936.

(54) Jacucci, G.; Schertel, L.; Zhang, Y.; Yang, H.; Vignolini, S. Light Management with Natural Materials: From Whiteness to Transparency. Adv. Mater. 2020, No. 2001215.

(55) Fischer, H. R. Micro-Thermal Analysis of Polyester Coatings. Appl. Surf. Sci. 2010, 256, 3714-3717.

(56) Romdhane, I. H.; Price, P. E.; Miller, C. A.; Benson, P. T.; Wang, S. Drying of Glassy Polymer Films. Ind. Eng. Chem. Res. 2001, 40, 3065-3075.

(57) Guerrier, B.; Bouchard, C.; Allain, C.; Bénard, C. Drying Kinetics of Polymer Films. AIChE J. 1998, 44, 791-798.

(58) Prakash, S. S.; Francis, L. F.; Scriven, L. E. Microstructure Evolution in Dry Cast Cellulose Acetate Membranes by Cryo-SEM. J. Membr. Sci. 2006, 283, 328-338.

(59) Wang, Y.; LeVan, M. D. Adsorption Equilibrium of Binary Mixtures of Carbon Dioxide and Water Vapor on Zeolites 5A and 13X. J. Chem. Eng. Data 2010, 55, 3189-3195.
(60) Wu, D.; Xu, F.; Sun, B.; Fu, R.; He, H.; Matyjaszewski, K. Design and Preparation of Porous Polymers. Chem. Rev. 2012, 112, 3959-4015.

(61) Mohamed, M. H.; Wilson, L. D. Porous Copolymer Resins: Tuning Pore Structure and Surface Area with Non Reactive Porogens. Nanomaterials 2012, 2, 163-186.

(62) Hjärtstam, J.; Hjertberg, T. Studies of the Water Permeability and Mechanical Properties of a Film Made of an Ethyl CelluloseEthanol-Water Ternary Mixture. J. Appl. Polym. Sci. 1999, 74, 20562062.

(63) Khare, V. P.; Greenberg, A. R.; Kelley, S. S.; Pilath, H.; Roh, I. J.; Tyber, J. Synthesis and Characterization of Dense and Porous Cellulose Films. J. Appl. Polym. Sci. 2007, 105, 1228-1236. 